\title{
ON A PROBLEMS RELATED TO A CONCEPT OF SOIL THERMAL DIFFUSIVITY AND ESTIMATION OF ITS DEPENDENCE ON SOIL MOISTURE
}

\author{
M.V. Glagolev ${ }^{1,2,3,4}$, A.F. Sabrekov ${ }^{1,2}$ \\ ${ }^{1}$ Yugra State University, Khanty-Mansyisk, Russia \\ 2 A.N. Severtsov Institute of Ecology and Evolution, Russian Academy of Sciences, Moscow, Russia \\ ${ }^{3}$ Lomonosov Moscow State University, Moscow, Russia \\ ${ }^{4}$ Institute of Forest Science, Russian Academy of Sciences, Uspenskoe (Moscow region), Russia
}

Corresponding authors: M.V. Glagolev, m_glagolev@mail.ru, A.F. Sabrekov, sabrekovaf@gmail.com

Citation: Glagolev MV, Sabrekov AF, 2019. On a problems related to a concept of soil thermal diffusivity and estimation of its dependence on soil moisture. Environmental Dynamics and Global Climate Change. 10:68-85. https://doi.org/10.17816/edgcc21202

Two problems in the theory of soil thermal conductivity are considered. First, the concept of the thermal diffusivity coefficient $(\kappa)$ is discussed. It was shown that this coefficient can be used for model predictions only in a certain special cases. In the general case (when the soil thermal capacity and thermal conductivity vary in space and/or in time), the thermal diffusivity does not naturally appear - see Eq. (9) and Table. It could be artificially introduced into the heat equation - see Eq. (10) - but, in any case, to solve this equation (i.e., to calculate the dynamics of the soil temperature), this one parameter is not sufficient. It is necessary to set both the heat capacity and thermal conductivity as functions of spatial and temporal coordinates or as functions of environmental factors (e.g. soil moisture) depending on these coordinates. In this regard, the widespread misconception of the supposed sufficiency of one parameter (soil thermal diffusivity as a ratio of soil thermal conductivity to thermal capacity) for solving the heat equation using numerical methods is discussed. The examples of the common difference schemes used in computational practice show that this is not the case.

Secondly, the condition number for the problem of parameters identification for the dependence of the soil thermal diffusivity coefficient on moisture for one well-known equation (4) is considered. The condition number $(\alpha)$ is the ratio of the relative error of the solution to the relative error of the input data - see Eq. (5). Because in practice the input data errors cannot be made randomly small, the accuracy of the solution is limited.

It is shown on real examples, the problem of parameters identification for the dependence of $\kappa$ on moisture is often ill-conditioned when solved by the least-squares method. The exact answer on the question about the value of $\alpha$, in which it should be recognized that the problem has become ill-conditioned, significantly depends on requirements of the solution accuracy. However it is usually considered for the problems like that $\alpha \gg 1$. In the considered examples the maximum values of $\alpha$ in some cases reached 10 or more. If accuracy of measurements for the $\kappa$ (or its variability for soils) is $\sim 10 \%$, the problem should be considered as ill-conditioned.

However, sometimes its stability can be significantly improved if simple constraints are set for certain parameters ("least-squares method with constraints"). Restrictions can be formulated based on purely logical considerations or additional data on soil physical properties. As an example of the latter, we can point out that the actual values of the volumetric soil moisture for mineral soils do not exceed $0.6-0.7 \mathrm{~cm}^{3} \mathrm{H}_{2} \mathrm{O} / \mathrm{cm}^{3}$ Soil. Therefore, the parameter $\theta_{0}$, which has the meaning of such a humidity at which the maximum thermal diffusivity is reached, cannot be greater than $0.7 \mathrm{~cm}^{3} \mathrm{H}_{2} \mathrm{O} / \mathrm{cm}^{3}$ Soil. The corresponding logical constraint is weaker: $\theta_{0} \leq 1$ (volumetric soil moisture $\theta>1$ has no meaning). Another obvious logical constraint can be derived from the fact that for $\theta=1$ we have pure water, therefore the parameters of $\kappa(\theta)$ must be such that for $\kappa(1)$ we have a numerical value of water thermal diffusivity coefficient.

The following simple "bootstrap" algorithm was used as a method for analyzing the conditionality of the parameter identification problem. Random noise $\left(\Delta \kappa_{\mathrm{i}}\right)$ simulating the uncertainty of experimental data $\left(\kappa_{\mathrm{i}}\right)$ was added into experimental data. The problem of parameters identification for Eq. (4) was solved based on the input data of $\left(\kappa_{i}+\Delta \kappa_{i}\right)$. This procedure was repeated $\mathrm{N}=\left(\mathrm{N}_{\mathrm{p}}+1\right) \cdot \mathrm{N}_{\mathrm{e}}$ times, where $\mathrm{N}_{\mathrm{p}}-$ number of identified parameters, $\mathrm{N}_{\mathrm{e}}$ - number of experimental points. As a result, $\mathrm{N}$ values of each identified parameter of the formula (4) were obtained, and on these sets of values statistical characteristics of the dispersion were calculated, which were compared with the error $\Delta \kappa$ of the original data.

Key words: soil temperature modeling, heat equation, soil thermal conductivity, ill-conditioned problems.

В работе рассматриваются две проблемы, возникающие в теории теплопроводности почв. Во-первых, обсуждается понятие коэффициента температуропроводности в свете того, что оно появляется только в отдельных весьма частных случаях, а в общем случае (когда теплоемкость и теплопроводность изменяются по пространству и/или с течением времени) коэффициент температуропроводности естественным образом вообще не возникает. Для такой среды с переменными (по пространству и во времени) свойствами он 
может быть искусственно введен в уравнение динамики температурного поля, но, в любом случае, для решения этого уравнения (т. е. для расчета динамики температурного поля) недостаточно одного параметра - необходимо задать и теплоемкость, и теплопроводность как функции пространственной и временной координат или как функции факторов среды (например, влажности), зависящих от этих координат. В связи с этим обсуждается и распространенное заблуждение о якобы достаточности одного параметра (коэффициента температуропроводности как отношения теплопроводности к теплоемкости) при решении вышеуказанного уравнения численными методами. На примерах основных разностных схем, применяемых в вычислительной практике, показано, что это не так.

Во-вторых, рассматривается число обусловленности задачи идентификации параметров одного известного уравнения зависимости коэффициента температуропроводности от влажности. На конкретных примерах показано, что данная задача при ее решении обычным методом наименьших квадратов часто является плохо обусловленной. Однако иногда ее обусловленность удается существенно улучшить при наложении простейших ограничений на искомые параметры (метод наименьших квадратов с ограничениями).

\section{BBEGEHИE}

\section{Используемые сокращения}

ПроСКРаС - пространственный «слой» конечно-разностной сетки;

$\mathrm{CV}$ - коэффициент вариации;

ICoP - плохо обусловленная задача (англ.: Ill-Conditioned Problem);

LeSP - задачи наименьших квадратов (англ.: Least Squares Problem);

TheD - коэффициент температуропроводности (англ.: Thermal Diffusivity [Lettau, 1971]);

ThePOS - термические (теплофизические) характеристики почвы (англ.: Thermal Properties Of Soil [Jury et al., 1991, p. 178]).

\section{Тепловой и температурный режимы почвы}

Температура почвы является важнейшим экологическим фактором, определяющим жизненные условия для высших растений (в том числе сельскохозяйственных культур) и почвенной фауны [Растворова, 1983, с. 168]. В частности, вязкость воды обратно пропорциональна температуре и при $4{ }^{\circ} \mathrm{C}$ она достигает величины, практически прекращающей поступление воды в растение. Температура почвы имеет большое значение и для жизнедеятельности микроорганизмов. Чувствительность микронаселения почвы к ее температуре столь значительна, что существует классификация микробов по их отношению к температуре (психрофильные, мезофильные, темотолерантные и термофильные микроорганизмы) [Ревут, 1972, с. 325, 327]. Контрасты температуры во времени (суточные и сезонные амплитуды температур) являются жестким «экологическим фильтром», ограничивающим состав и активность почвенной биоты [Растворова, 1983, с. 168].

Тепловой режим - это процессы поступления, перераспределения и расхода тепла в почве и на ее границах. А порождаемую этими процессами динамику температуры на поверхности и в различных слоях почвы называют температурным режимом почвы [Шеин,
2005 , с. 330]. Температурный режим является существенным фактором почвообразования [Ревут, 1972, с. 328]. Достаточно напомнить, что функцией от температуры являются скорость химических реакций, физические свойства вещества, слагающего почву (вязкость и поверхностное натяжение почвенного раствора, электропроводность и др.), а также испарение, диффузия и многие другие элементарные почвенные процессы. Перепады температур по профилю почвы (градиенты температур) имеют большое значение для влагопереноса в почве, поскольку парообразная и жидкая влага передвигаются от более нагретых участков к более холодным. Кроме того, колебания температуры и связанные с ними объемные изменения жидкой и газообразной фазы являются важным фактором воздухообмена между почвенным и атмосферным воздухом [Растворова, 1983, с. 168-169].

Таким образом, температура почвы является одним из решающих факторов в таких важнейших процессах, как образование и разрушение органических веществ в круговороте элементов и соединений на нашей планете [Ревут, 1972, с. 328], а теплофизика почвы - одна из важных отраслей технической и агрономической физики [Чудновский, 1976, с. 6], более того, по мнению И.Б. Ревута [1972, с. 329], изучение температуры почвы следует признать важнейшей задачей физики почв.

\section{Математическое моделирование в почвенной теплофизике}

Совокупность задач, дающих теоретическое описание теплового режима почв; оценку факторов, влияющих на его динамику; обнаружение причин, приводящих к его изменениям при разнообразных обстоятельствах, является главным в науке о теплообмене в почве. Генеральной задачей проблемы в целом является умение находить, анализировать, научно предвидеть и даже регулировать все элементы теплового режима почв [Чудновский, 1976, с. 7]. 
Если мы знаем распределение температуры по профилю почвы, то появляется возможность рассчитать распределение температуры на следующий момент времени, т. е. осуществить прогноз [Шеин, 2005, с. 327]. Такой прогноз нужен как для практической агрофизики, так и при проведении фундаментальных исследований в области почвоведения, в частности в условиях глобального изменения климата (учитывая, что любые существующие обоснованные сценарии этого изменения в первую очередь говорят об изменении температуры).

В качестве примера практических приложений достаточно упомянуть, что математические модели могут быть полезными при построении алгоритмов программирования теплового режима сельскохозяйственного поля. В частности, необходимость построения математических моделей, описывающих процессы формирования теплового режима в среде обитания растений, возникает при проведении тепломелиоративных мероприятий [Куртенер и Чудновский, 1979, с. 3-4]. Теоретические приложения чрезвычайно многообразны и затрагивают моделирование практически любых явлений, связанных с почвой, в основе которых лежат зависящие от температуры микробиологические, химические и физические процессы [Bowden et al., 1974; Groenevelt, 1974; Sabrekov et al., 2013; Zhu et al., 2013].

Почва представляет собой трехфазную систему, состоящую из остова-скелета, представляющего совокупность огромного количества твердых частиц разнообразной формы и величины, разделенных промежутками, заполненными газом, влагой или и тем и другим одновременно. В общем случае теплообмен в такой системе осуществляется путем:

1) теплопроводности вдоль отдельной частицы - элемента твердого скелета материала;

2) молекулярной теплопроводности в среде, заполняющей промежутки между частицами;

3) теплообмена на границах систем «твердая частица-газ» и «твердая частица-жидкость» ${ }^{1}$;

4) излучения;

5) конвекции газа и влаги, содержащихся в порах.

Очевидно, что расчет температурного поля в почве, где все процессы взаимосвязаны, задача исключительно сложная. Эффективным средством, позволяющим успешно решать мно-

${ }^{1}$ В цитируемом нами первоисточнике - [Куртенер и Чудновский, 1979, с. 12] - теплообмен на границе системы «твердая частица-жидкость» не упомянут, но представляется, что он должен иметь место. Мы благодарны анонимному рецензенту, указавшему нам на это. гие практические задачи, может служить модель эквивалентной теплопроводности почвы [Куртенер и Чудновский, 1979, с. 12].

Первое упрощение, которое делается при исследовании температурного поля почвы, состоит в возможности принятия его одномерным. Это значит, что величина температуры $(T)$ считается изменяющейся лишь вдоль глубины почвы $(z)$; горизонтальные поверхности, параллельные наружной поверхности почвы, принимаются изотермическими, а поток тепла направлен по нормали к последним от мест с большей температурой к местам с наименьшей температурой [Нерпин и Чудновский, 1967, с. 231]. Уравнение сохранения для количества тепла имеет вид

$$
\partial Q / \partial t+\partial q / \partial z+r=0,
$$

где $Q$ - количество тепла (тепловой энергии) в единице объема почвы (Дж/м $\left.{ }^{3}\right) ; t-$ время (c) [Jury et al., 1991, p. 178]; $q$ - плотность потока тепла, который представляет собой скорость переноса тепла через единицу площади $\left(\right.$ Дж $\cdot \mathrm{M}^{-2} \cdot \mathrm{c}^{-1}$ ) [Воронин, 1986, с. 221]; $r$ - мощность источника или стока тепла в единице объема почвы (Дж $\left.\cdot \mathrm{M}^{-3} \cdot \mathrm{c}^{-1}\right)$, обусловленная физическими, химическими и биохимическими процессами (тепло может выделяться или поглощаться при адсорбции/десорбции, таянии/ замерзании, а также в результате химических реакций и жизнедеятельности организмов). Здесь ради простоты мы будем предполагать, что $r \approx 0$, поскольку для существа рассматриваемых нами вопросов величина $r$ не имеет особого значения.

Учитывая, что количество тепла в объеме почвы линейно зависит от температуры $(T)$ [Jury et al., 1991, p. 178], легко получить уравнение $^{2}$, описывающее динамику температуры почвы:

$$
\partial\left(\rho \cdot C_{\mathrm{m}} \cdot T\right) / \partial t=-\partial q / \partial z,
$$

где $\rho-$ плотность почвы (кг/м $\left.{ }^{3}\right) ; C_{\mathrm{m}}-$ удельная теплоемкость почвы (Дж $\left.\mathrm{K \Gamma}^{-1} \cdot \mathrm{K}^{-1}\right)$.

Второе упрощающее предположение, которое делается в идеализированной постановке задачи, заключается в том, что почва рассматривается как квазиоднородное тело, теплофизические свойства которого описываются

2 Некоторые авторы (см., например, [Нерпин и Чудновский, 1967, с. 240; Воронин, 1986, с. 221-223, Шеин, 2005, с. 326]) пишут, что, согласно уравнению неразрывности, в отсутствие любых источников или стоков тепла $\rho \cdot C_{\mathrm{m}} \cdot \partial T / \partial t=\partial q / \partial z$. Обращаем внимание читателей, что знаки перед производными в правой и левой частях правильного уравнения должны быть разными, как это имеет место в уравнении (1). 
некоторыми эффективными величинами [Куртенер и Чудновский, 1979, с. 12]. При этом принимается справедливость закона 3 о прямой пропорциональности между плотностью теплового потока и градиентом температуры [Нерпин и Чудновский, 1967, с. 232]:

$$
q=-\lambda \cdot \partial T / \partial z
$$

Подставляем в (1) вместо $q$ его значение в соответствии с уравнением Фурье:

$$
\partial\left(\rho \cdot C_{\mathrm{m}} \cdot T\right) / \partial t=\partial(\lambda \cdot \partial T / \partial z) / \partial z
$$

где $\lambda-$ коэффициент теплопроводности $\left(\mathrm{BT} \cdot \mathrm{M}^{-1} \cdot \mathrm{K}^{-1}\right)$ [Шеин, 2005, с. 326] или, более точно, коэффициент кондуктивной теплопроводности почвы [Нерпин и Чудновский, 1967, c. 232]. Обычно считают, что $\rho \cdot C_{\mathrm{m}}=$ const, поэтому в литературе по физике почв последнее уравнение чаще всего (см., например, [Нерпин и Чудновский, 1967, с. 233; Куртенер и Чудновский, 1979, с. 12; Воронин, 1986, с. 222; Калюжный и Лавров, 2012]) можно встретить в следующем виде ${ }^{4}$ :

$$
\rho \cdot C_{\mathrm{m}} \cdot \partial T / \partial t=\partial(\lambda \cdot \partial T / \partial z) / \partial z .
$$

\section{Температуропроводность почвы}

Относительно последнего уравнения в своем учебнике по физике почв Воронин [1986, c. 222] писал: «Для того, чтобы определить... изменения $T$ во времени... на заданной глубине, это уравнение следует представить в следующем виде: $\partial T / \partial t=\left[\lambda /\left(\rho \cdot C_{\mathrm{m}}\right)\right] \cdot \partial^{2} T / \partial z^{2} »$.

В результате появился новый коэффициент $\lambda /\left(\rho \cdot C_{\mathrm{m}}\right)=\kappa\left(\mathrm{M}^{2} / \mathrm{c}\right)$, состоящий из известных нам параметров, который называют температуропроводностью5 ${ }^{5}$. Заметим, что ее размерность совпадает с коэффициентом диффузии, поэтому нередко величину к называют термо-

3 Шеин [2005, с. 323] и Jury et al. [1991, p. 174] называют его «законом Фурье» («Fourier's law»), а Нерпин и Чудновский [1967, с. 232] - «законом Фика». Последнее, на наш взгляд, неверно, поскольку понятие «закон Фика» прочно утвердилось (см., например, [Ревут, 1972, с. 196-197; Орлов и др., 1987, с. 120; Jury et al., 1991, p. 203; Brady, 1995, p. 270]) за линейной связью между плотностью потока массы и градиентом конщентрации.

${ }^{4}$ Или в очень похожем, когда произведение $\rho \cdot C_{\mathrm{m}}$ заменяют одним параметром - объемной теплоемкостью почвы $C=\rho \cdot C_{\mathrm{m}}$ (англ.: soil volumetric heat capacity [Jury et al., 1991, p. 178], тогда как $C_{\mathrm{m}}-$ specific heat [Lettau, 1971]).

5 Точнее говоря, Е. Шеин [2005, с. 326] называет температуропроводностью коэффициент $\lambda / \rho \cdot C_{\mathrm{m}}$. Конечно, это - очевидная ошибка или опечатка: согласно правилам приоритета математических операций, $\lambda / \rho \cdot C_{\mathrm{m}}=(\lambda / \rho) \cdot C_{\mathrm{m}}=C_{\mathrm{m}} \cdot(\lambda / \rho) \neq \lambda /\left(\rho \cdot C_{\mathrm{m}}\right)$. диффузивностью ${ }^{6}$ [Шеин, 2005, с. 326-327]. Итак, мы пришли к относительно простому уравнению

$$
\partial T / \partial t=\kappa \cdot \partial^{2} T / \partial z^{2}
$$

Тогда для решения проблем почвенной теплофизики - для решения уравнения при различных краевых условиях, необходимо располагать данными о теплофизических характеристиках в зависимости от природы и внутренних особенностей почвенного материала: от влажности, плотности, дисперсности, химикоминералогического состава и т. д. [Чудновский, 1976, с. 7-8]. По свидетельству А.Ф. Чудновского [1976, с. 8], эти данные для большинства почв Советского Союза в основном уже были получены им и его учениками к середине 70-х гг. XX-го в. Тем не менее, такая работа продолжалась и после этого - см., например, [Набиев и Гусейнов, 1990; Гаврильев, 2004; Федотов и Жуков, 2004; Лапина, 2019; Лебедев и др., 2019]. В частности, для зависимости TheD от влажности ( $\theta)$ T.A. Архангельской [2004] была предложена эмпирическая формула вида

$$
\kappa=\kappa_{0}+a \cdot \exp \left(-0.5 \cdot \ln ^{2}\left(\theta / \theta_{0}\right) / b^{2}\right),
$$

где $\kappa_{0}-$ The D сухой почвы; $a-$ разность между максимальной и минимальной температуропроводностями почвы; $\theta_{0}-$ влажность,

6 Часто к называют «коэффициентом температуропроводности» (TheD) [Набиев и Гусейнов, 1990; Федотов и Жуков, 2004; Лапина, 2019]. Также Шеин [2005, с. 328] указывает, что в зарубежной литературе $\kappa$ называют коэффициентом термодиффузии. Поскольку для обоснования этого никаких ссылок не приводится, мы не можем проверить справедливость данного утверждения. Но, скорее всего, неверная трактовка возникла просто в результате некорректного перевода. Обычно коэффициентом термодиффузии (англ.: coefficient of thermodiffusion [Costesèque et al., 2011]) называют другую величину.

Вообще, термодиффузия - это перенос компонент газовой смеси или растворов при наличии в них градиента температуры. Если разность температур поддерживается постоянной, то вследствие термодиффузии в объеме смеси возникает градиент концентрации, что вызывает и обычную диффузию. В бинарной смеси при постоянном давлении и отсутствии внешних сил полный диффузионный поток вещества $\boldsymbol{j}_{i}=n \cdot D \cdot \operatorname{grad}\left(c_{i}\right)-n \cdot(D \mathrm{~T} / T) \cdot \operatorname{grad}(T)$, где $D-$ коэффициент диффузии, $n-$ число частиц смеси в единице объема, $c_{i}-$ концентрация $\left(c_{i}=n_{i} / n ; i=1,2\right), D_{\mathrm{T}}-$ коэффициент термодиффузии ( $D_{\mathrm{T}}$ сильно зависит от межмолекулярного взаимодействия) [Зубарев, 1998]. В таком же смысле термин «coefficient of thermodiffusion» (или «коэффициент термической диффузии») употребляется, например, в [Grew and Ibbs, 1952; Котоусов, 1973; Costesèque et al., 2011]. 
при которой достигается максимум температуропроводности; $b$ - параметр формы кривой (характеризует ширину пика).

\section{Обусловленность вычислительной задачи}

Пусть задача корректна (ее решение существует, единственно и устойчиво по входным данным). Теоретически, наличие у задачи устойчивости означает, что ее решение может быть найдено со сколь угодно малой погрешностью, если погрешности исходных данных достаточно малы. Однако на практике погрешности входных данных не могут быть сделаны сколь угодно малыми - точность их ограничена. Под обусловленностью вычислительной задачи понимают чувствительность ее решения к малым погрешностям входных данных. Задачу называют плохо обусловленной (далее: ICoP - от англ. ill-conditioned problem), если малым погрешностям входных данных $(\delta f)$ отвечают сильные изменения решения $(\delta v)$. Величина $\alpha$ в неравенстве $\delta v \leq \alpha \cdot \delta f$ называется относительным числом обусловленности (если $\delta v$ и $\delta f-$ именно относительные погрешности), причем в этом неравенстве может фигурировать граница погрешности, т. е.

$$
\delta v=\alpha \cdot \delta f .
$$

Для ICoP $\alpha \gg 1$. Но точный ответ на вопрос о значении $\alpha$, при котором следует признать, что задача стала плохо обусловленной, существенно зависит от предъявляемых требований к точности решения и от уровня обеспечиваемой точности исходных данных [Амосов и др., 2008, c. 54-55]. Кроме того, для нелинейных моделей следует ожидать $\alpha \neq$ const, поэтому задача может быть хорошо обусловленной при одних значениях $\delta f$, но становиться ICоР при других (обычно - больших) $\delta f$ [Глаголев и Сабреков, 2019]. Плохо обусловленными часто являются задачи определения (по экспериментальным данным) параметров какой-либо математической модели. В этом случае формальное решение, конечно, может быть найдено (например, при помощи метода наименьших квадратов), однако оно, подчас, содержит столь большую погрешность, что, фактически, никакого отношения к реальности не имеет. Определить обусловленность (т. е. найти число обусловленности) какой-либо конкретной задачи можно при помощи различных методов, как аналитических, так и численных.

Среди последних выделим группу так называемых «методов статистического анализа чувствительности» [Полак и др., 1984, с. 158]. Повидимому, из них наиболее простым, но весьма эффективным является алгоритм, основанный на так называемой «bootstrap»-процедуре, которая будет описана ниже. Этот метод анализа чувствительности идентификации параметров модели к погрешностям экспериментальных данных уже неоднократно применялся, в том числе в математических задачах биологии и почвоведения - см., например, [Еремеев и др., 1989, Panikov et al., 1992; Глаголев и др., 2010, c. 27-31, 94-101; Моченов и др., 2017].

В представляемой вниманию читателей работе нашей целью было: рассмотреть некоторые проблемы, возникающие в связи с понятием температуропроводности почв и ее вычислением. В рамках данной цели ставились две задачи: во-первых, определить обусловленность нескольких конкретных задач идентификации параметров зависимости коэффициента температуропроводности от влажности (в тех ситуациях, когда температуропроводность, все-таки, имеет смысл) и, во-вторых, выделить те ситуации, когда понятие температуропроводности становится бесполезным для практических вычислений.

\section{METOQ}

Идея, лежащая в основе одного из способов анализа чувствительности, весьма проста и имеет общий характер: решить задачу с несколькими разными данными и посмотреть, насколько чувствительно решение к изменению в данных [Rice, 1981]. Если речь идет об обработке экспериментальных данных, то, в идеале, данные для анализа чувствительности каждый раз следовало бы получать из эксперимента. Однако в ряде случаев это невозможно (например, эксперимент слишком дорог или обрабатываются данные экспериментальных измерений, ранее опубликованных в литературе).

В качестве экспериментальных данных мы использовали значения, снятые с графиков температуропроводности, опубликованных в [Архангельская, 2009; Лебедев и др., 2019]. Таким образом, не было возможности получить какие-либо дополнительные данные, сверх тех, что уже были представлены в указанных работах. В такой ситуации остается воспользоваться «bootstrap»-методом.

Процедура «bootstrap» позволяет произвести оценку по данным единственной выборки. Идея состоит в имитации процесса получения многих выборок такого же объема, как и исходная выборка. Таким образом, располагая лишь одной выборкой, мы можем генерировать необходимое число других выборок, адекватных тем, которые в реальных условиях можно было бы получить случайным образом [Diaconis and Efron, 1983]. 
Краткое $^{7}$ формальное описание алгоритма анализа обусловленности задачи идентификации параметров ( $\kappa_{0}, a, b$ и $\left.\theta_{0}\right)$ зависимости коэффициента температуропроводности от влажности таково:

(1) $i=1$;

(2) на измеренные в эксперименте значения TheD (составляющие в совокупности всю выборку, содержащую $N_{f}$ элементов:

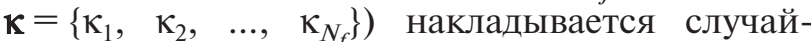
ный шум $\Delta \boldsymbol{\kappa}_{j}=\left\{\Delta \kappa_{1}, \Delta \kappa_{2}, \ldots, \Delta \kappa_{N_{f}}\right\}$, характеризуюшийся коэффициентом вариации CV (тем самым моделируется другая выборка, которую можно было бы получить, если поставить новый эксперимент по измерению TheD);

(3) по зашумленным значениям $\left(\boldsymbol{\kappa}+\Delta \boldsymbol{\kappa}_{j}\right)$ путем решения обратной задачи (методом наименьших квадратов) рассчитывается вектор параметров $\boldsymbol{v}=\left\{\kappa_{0}, a, b, \theta_{0}\right\}$ и его элементы записываются в векторы $\mathbf{K}, \mathbf{A}, \mathbf{B}$ и $\mathbf{O}$ в качестве $i$-ых элементов: $\mathbf{K}_{i}=\kappa_{0}, \mathbf{A}_{i}=a, \mathbf{B}_{i}=b$ и $\mathbf{O}_{i}=\theta_{0}$;

(4) $i=i+1$;

(5) если $i \leq N$, то перейти к п. (2);

(6) вычислить коэффициенты вариации по всем элементам векторов $\mathbf{K}, \mathbf{A}, \mathbf{B}$ и $\mathbf{O}$ : $\mathrm{CV}_{\mathrm{K}}=\operatorname{std}(\mathbf{K}) / \operatorname{mean}(\mathbf{K}), \quad \mathrm{CV}_{a}=\operatorname{std}(\mathbf{A}) / \operatorname{mean}(\mathbf{A})$, $\mathrm{CV}_{b}=\operatorname{std}(\mathbf{B}) / \operatorname{mean}(\mathbf{B}), \mathrm{CV}_{\theta_{0}}=\operatorname{std}(\mathbf{O}) / \operatorname{mean}(\mathbf{O}) ;$

(7) вычислить соответствующие числа обусловленности: $\alpha_{\kappa}=\mathrm{CV}_{\kappa} / \mathrm{CV}, \quad \alpha_{a}=\mathrm{CV}_{a} / \mathrm{CV}$, $\alpha_{b}=\mathrm{CV}_{b} / \mathrm{CV}, \alpha_{\theta_{0}}=\mathrm{CV}_{\theta_{0}} / \mathrm{CV}$;

(8) КОНЕЦ.

Значение $N$ в описанном алгоритме выбирали согласно [Варфоломеев и Гуревич, 1999, с. 478]: $N=\left(N_{\mathrm{v}}+1\right) \cdot N_{\mathrm{f}}$, где $N_{\mathrm{v}}-$ количество идентифицируемых параметров (в данном случае $\left.N_{\mathrm{v}}=4\right)$. Данный алгоритм выполнялся для различных значений $\mathrm{CV}$, что позволяло понять всю картину обусловленности задачи (и при малых, и при больших погрешностях исходных данных).

\section{PEBYกலTATbI}

Если задачу идентификации параметров зависимости (4) для горизонта Аһ (Владимирское ополье) по данным [Архангельская, 2009,

7 Здесь мы позволили себе ограничиться кратким описанием, но общепринятое требование возможности полного воспроизведения использованной методики выполнено благодаря тому, что в Приложении мы приводим текст соответствующей программы для ЭВМ. Однако эта программа написана с прицелом на задачи анализа обусловленности любой одномерной положительной зависимости с любым числом параметров и поэтому несколько отличается от приведенного конкретного алгоритма, ориентированного на анализ обусловленности задачи идентификации четырех параметров. pис. 1б] ( $n=11$ значений к, полученных в эксперименте) рассматривать как чисто математический пример использования метода наименьших квадратов, то числа обусловленности $(\alpha)$ будут весьма сильно различаться для разных параметров. При CV исходных данных $1 \div 32 \%$ для $\kappa_{0}, a, b$ и $\theta_{0}$ были получены, соответственно, значения $\alpha: 0.7 \div 1.4,5 \div 13,4 \div 8$ и $9 \div 25$.

Однако, если опираться на указанный выше физический смысл $\theta_{0}$, то возможная величина этого параметра должна быть принудительным образом ограничена (хотя бы из чисто логических соображений следует, что - по определению влажность не может быть более $1 \mathrm{~cm}^{3} \mathrm{H}_{2} \mathrm{O} / \mathrm{cm}^{3}$ почвы; но обычно есть и более точная информация об объекте, например, реальные значения объемной влажности минеральных почв не превышают 0.6-0.7 $\mathrm{cm}^{3} \mathrm{H}_{2} \mathrm{O} / \mathrm{cm}^{3}$ почвы). Кроме того из физического смысла к ${ }_{0}$ и $a$ очевидно, что эти параметры - положительные. Решение задачи наименьших квадратов (LeSP) с такими ограничениями приводит уже к иному результату: при небольших CV исходных данных $(1 \div 8 \%)$, когда наложенное ограничение «срабатывает» редко, значения $\alpha$ по сравнению с предыдущим вариантом расчета без ограничения изменяются не сильно. Соответственно, имеем $\alpha$ для $\kappa_{0}, a, b$ и $\theta_{0}: 0.6 \div 0.8,3 \div 5,5 \div 7$ и $5 \div 10$. Но при больших CV исходных данных $(10 \div 32 \%)$ независимо от них CV идентифицируемых параметров не могут расти из-за наложенного ограничения, поэтому числа обусловленности падают: $2 \div 3$, $2 \div 4$ и $1 \div 4$, соответственно, для $a, b$ и $\theta_{0}$ (для $\kappa_{0}$ число обусловленности было мало и таковым оно остается: $\alpha_{\kappa_{0}}=0.6 \div 0.9$ ).

При идентификации параметров зависимости (4) для горизонта ЕВ (Владимирское ополье) по данным [Архангельская, 2009, рис. 1Е] $(n=8)$ число обусловленности для $\kappa_{0}$ во всем исследованном диапазоне $\mathrm{CV}$ исходных данных $(1 \div 32 \%)$ находится в границах от 0.6 до 1.4 ; для $a-$ в границах от 1.2 до 3 ; для $b-$ от 2.8 до 4.7; и, наконец, для $\theta_{0}-1.9 \div 5.1$ (за исключением случая $\mathrm{CV}=32 \%$, когда $\alpha_{\theta_{0}}=12$ ). Разумеется, такой результат относится только к случаю решения LeSP с ограничением. Если решать обычную задачу наименьших квадратов (т. е. без каких-либо априорных ограничений), то некоторые числа обусловленности становятся порядка десятков и задача идентификации, следовательно, плохо обусловлена.

Теперь рассмотрим пример с существенно большим количеством экспериментальных точек $(n=70)$ - идентификацию параметров зависимости (4) для горизонта $\mathrm{A}_{\text {пахII }}$ (пахотного горизонта, подстилаемого минеральным подпахотным горизонтом) по данным [Архангельская, 2009, рис. 2б]. Несмотря на существенное 
а) для примеров из [Архангельская, 2009]

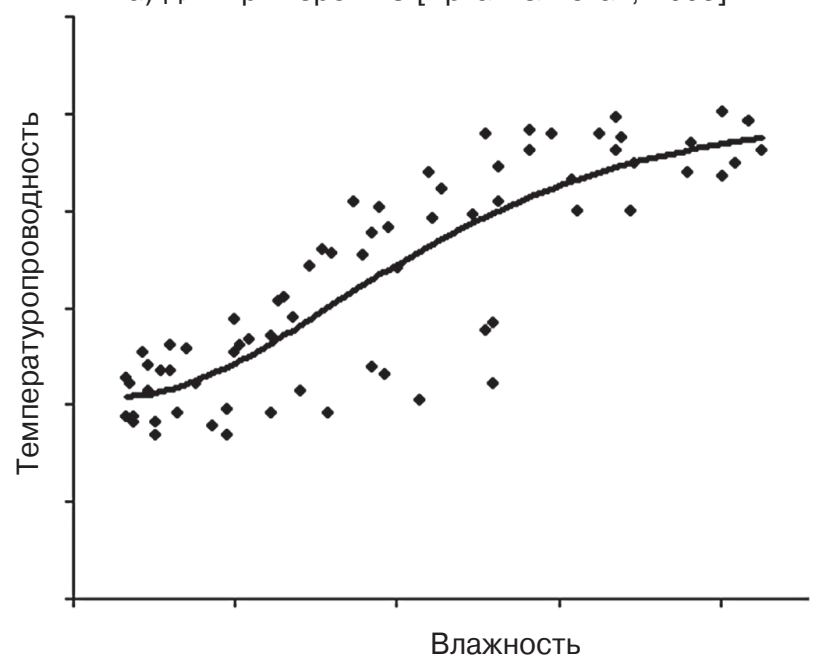

b) для примера из [Лебедев и др., 2019]

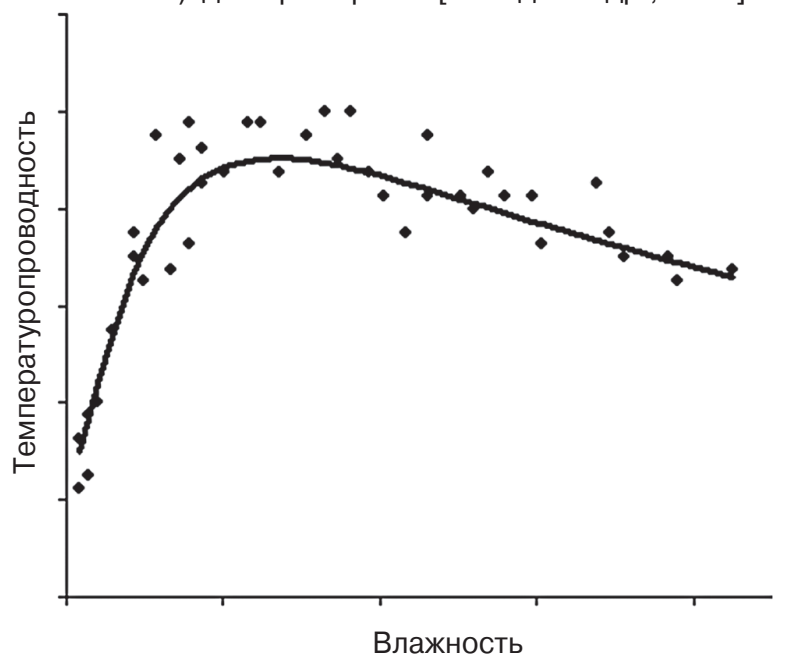

Pnc. 1. Типичный вид зависимости коэффициента температуропроводности от влажности

Fis. 1. Typical type of dependence of the coefficient of thermal diffusivity on moisture

увеличение количества точек, картина решения изменилась мало. Число обусловленности для $\kappa_{0}$ во всем исследованном диапазоне CV исходных данных $(1 \div 30 \%)$ находится в границах $0.3 \div 0.4$ и $0.3 \div 0.5$, соответственно, при решении LeSP с ограничением или без. При небольших CV исходных данных $(1 \div 8 \%)$ число обусловленности для $a$ составляет около 2 , а при $10 \%<\mathrm{CV} \leq 30 \%$ - падает до 1 или возрастает до $4 \div 5$, соответственно, если решать LeSP с ограничением или без. Аналогично, число обусловленности для $\theta_{0}$ при CV исходных данных $1 \div 8 \%$ составляет около 3 , а при $10 \%<\mathrm{CV} \leq 30 \%$ - падает до 2 или возрастает до $5 \div 11$, соответственно, в случае LeSP с ограничением или без. Число обусловленности для $b$ во всех случаях составляет $1 \div 2$. Например, для LeSP без ограничения при CV исходных данных $10 \% \alpha_{\kappa_{0}} \approx 0.36, \alpha_{a} \approx 1.7, \alpha_{b} \approx 2.0$, $\alpha_{\theta_{0}} \approx 3.2 ;$ а при $\mathrm{CV}=20 \% \quad \alpha_{\kappa_{0}} \approx 0.41, \alpha_{a} \approx 4.4$, $\alpha_{b} \approx 2.1, \alpha_{\theta_{0}} \approx 9.3$.

Наконец, проведем идентификацию параметров зависимости (4) для рыхлых песков серогумусовых песчаных почв Воронежской области по экспериментальным данным из [Лебедев и др., 2019, рис. 2а]. Количество экспериментальных данных в этом примере $(n=41)$ почти в 2 раза меньше, чем в предыдущем, но обусловленность задачи оказывается несколько лучше. Во всем диапазоне $1 \%<\mathrm{CV} \leq 32 \%$ числа обусловленности для $a, b$ и $\theta_{0}$ находятся, соответственно, в интервалах $0.6 \div 0.9,1.0 \div 1.3$ и $0.4 \div 0.7$, и только для $\alpha_{\kappa_{0}}$, хотя и не достигает максимальных значений из предыдущих примеров, но существенно больше: $2 \div 7$ (в частности, при CV исходных данных $10 \% \alpha_{\kappa_{0}} \approx 3.1, \alpha_{a} \approx 0.7$, $\alpha_{b} \approx 1.1, \alpha_{\theta_{0}} \approx 0.4$ ).

\section{ОБСУЖА태트}

\section{Обусловленность задачи идентификации параметров}

К сожалению, ни в [Архангельская, 2009], ни в [Лебедев и др., 2019] не указано - какова была точность измерений к. Однако, по рис. 1 можно предположить, что в данном случае разброс экспериментальных данных (который составляет, как представляется, до 20\%), повидимому, определялся не столько инструментальной погрешностью физических измерений (в теплофизических измерениях она составляет, вероятно, 5-10\% $)^{8}$, сколько естественной природной вариабельностью образцов почвы. Впрочем, раз мы рассматриваем задачу определения параметров $\kappa_{0}, a, b$ и $\theta_{0}$, то для нас основной интерес имеет их погрешность, а не погрешность к. Но понятно, что последняя в значительной мере определяет точность вычисления указанных параметров. К сожалению, значения этих параметров, найденные Лебедевым и др. [2019], приводятся авторами без явного указания какой-либо характеристики разброса (погрешности).

Как известно, если число приводится в качестве результата без указания значения погрешности, то принято считать, что все его значащие цифры являются верными [Амосов и др., 2008, с. 31]. Лебедев и др. [2019] приводят значения параметров $\left(\kappa_{0}=2.507 \cdot 10^{-7} \mathrm{M}^{2} / \mathrm{c}\right.$, $a=6.533 \cdot 10^{-7} \mathrm{M}^{2} / \mathrm{c}, b=1.195$ и $\left.\theta_{0}=0.067\right)$, Как видим, с точностью до четырех значащих цифр (за исключением $\theta_{0}$ ). Значащая цифра в послед-

8 Мы благодарим одного из анонимных рецензентов за информацию о точности теплофизических измерений. 
нем разряде будет верной, если погрешность составляет чуть меньше половины последнего разряда. Таким образом, следует считать, что относительные погрешности ( $\delta$ ) найденных параметров:

$$
\begin{aligned}
& \delta_{\mathrm{\kappa}_{0}} \approx\left(5 \cdot 10^{-4} / 2.507\right) \cdot 100 \% \approx 0.02 \%, \\
& \delta_{a} \approx\left(5 \cdot 10^{-4} / 6.533\right) \cdot 100 \% \approx 0.008 \%, \\
& \delta_{b} \approx\left(5 \cdot 10^{-4} / 1.195\right) \cdot 100 \% \approx 0.04 \%, \\
& \delta_{\theta_{0}} \approx\left(5 \cdot 10^{-4} / 0.067\right) \cdot 100 \% \approx 0.7 \% .
\end{aligned}
$$

Однако проведенный нами выше анализ обусловленности приводит к совершенно другим результатам. Если (на основании рис. 1b) принять, что $\delta_{\kappa} \sim 10 \%$, то из формулы (5) имеем $\delta_{\mathrm{k}_{0}} \approx 3.1 \cdot 10 \% \approx 31 \%, \delta_{a} \approx 0.7 \cdot 10 \% \approx 7 \%$, $\delta_{b} \approx 1.1 \cdot 10 \% \approx 11 \%, \quad \delta_{\theta_{0}} \approx 0.4 \cdot 10 \% \approx 4 \%$. Как видно из рис. 2, эти оценки оказались весьма реалистичными: кривые, построенные по максимальным и минимальным значениям параметров как раз идут по границам «облака» экспериментальных точек.

В [Архангельская, 2009] даны какие-то характеристики разброса, но, к сожалению, автор не сообщает - какие (или мы оказались не в состоянии обнаружить эту информацию). Например, для горизонта $\mathrm{A}_{\text {пахII }}$ автор приводит следующие значения параметров:

$$
\begin{aligned}
& \kappa_{0}=(2.15 \pm 0.18) \cdot 10^{-7} \mathrm{M}^{2} / \mathrm{c}, \\
& a=(2.79 \pm 0.59) \cdot 10^{-7} \mathrm{M}^{2} / \mathrm{c}, \\
& b=0.78 \pm 0.25 \text { и } \theta_{0}=0.52 \pm 0.20 .
\end{aligned}
$$

Не зная, что в точности представляют собой данные характеристики разброса, можно записать в общем виде:

$$
\begin{aligned}
& \delta_{\mathrm{k}_{0}}=(A \cdot 0.18 / 2.15) \cdot 100 \% \approx A \cdot 8.4 \%, \\
& \delta_{a}=(A \cdot 0.59 / 2.79) \cdot 100 \% \approx A \cdot 21 \%, \\
& \delta_{b}=(A \cdot 0.25 / 0.78) \cdot 100 \% \approx A \cdot 32.1 \%, \\
& \delta_{\theta_{0}}=(A \cdot 0.2 / 0.52) \cdot 100 \% \approx A \cdot 38.5 \%,
\end{aligned}
$$

где $A$ - некоторый коэффициент. Как видим, согласно Архангельской [2009] $\delta_{\kappa_{0}}<\delta_{a}<\delta_{b}<\delta_{\theta_{0}}$, т. е. точнее всего определяется $\kappa_{0}$; $a$ вычисляется с относительной погрешностью в $(A \cdot 32.1 \%) /(A \cdot 8.4 \%) \approx 2.5$ раза большей; погрешность $b$ еще больше - она превышает $\delta_{\mathrm{k}_{0}}$ в $(A \cdot 21 \%) /(A \cdot 8.4 \%) \approx 3.8$ раза и, наконец, $\theta_{0}$ определяется из рук вон плохо, ибо погрешность больше чем $\delta_{\mathrm{k}_{0}}$ в $(A \cdot 38.5 \%) /(A \cdot 8.4 \%) \approx 4.6$ раза.

В случае нашего метода расчета результат зависит от величины CV исходных данных. При небольших погрешностях данных, получаем качественно такую же картину, как и Архангельская [2009]. Например, при CV исходных данных $10 \% \alpha_{\kappa_{0}}<\alpha_{a}<\alpha_{b}<\alpha_{\theta_{0}}$. Но представляется, что данные Архангельской [2009] характеризуются существенно большими значениями CV (около $20 \%$ - см. рис. 1а). Для таких больших CV средние члены ряда меняются местами: $\alpha_{\kappa_{0}}<\alpha_{b}<\alpha_{a}<\alpha_{\theta_{0}}$. В данном случае мы оперируем величинами $\alpha$, а не $\delta$, но по форму-

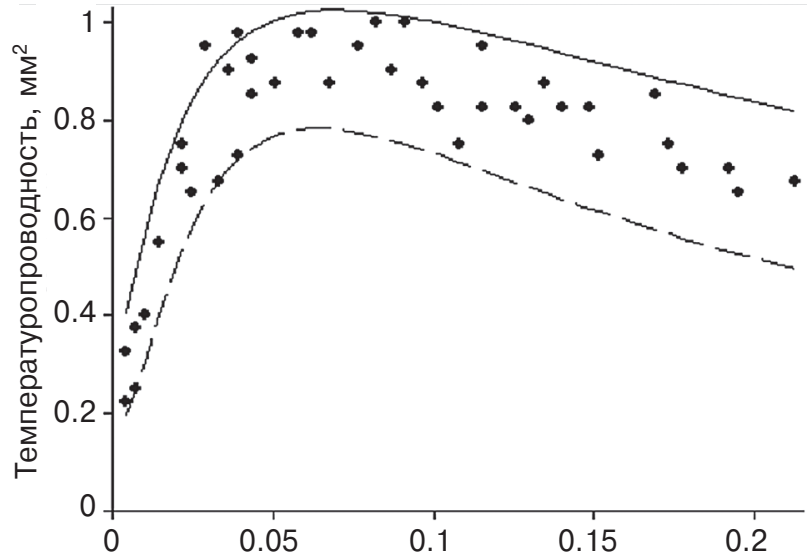

Pnc. 2. Разброс температуропроводностей, рассчитанных для предельно допустимого диапазона параметров

Fis. 2. Variation of temperature conditions calculated for the maximal range of parametres

• - экспериментальные данные из [Лебедев и др., 2019];

- - - расчет по (4) при

$\kappa_{0}^{\min }=\kappa_{0} \cdot\left(1-\delta_{\kappa_{0}} / 100 \%\right)=0.1728 \mathrm{MM}^{2} / \mathrm{c}$,

$a^{\min }=a \cdot\left(1-\delta_{a} / 100 \%\right)=0.6099 \mathrm{MM}^{2} / \mathrm{c}$,

$b^{\min }=b \cdot\left(1-\delta_{b} / 100 \%\right)=1.064$,

$\theta_{0}^{\min }=\theta_{0} \cdot\left(1-\delta_{\theta_{0}} / 100 \%\right)=0.064$

- - расчет по (4) при

$\kappa_{0}^{\max }=\kappa_{0} \cdot\left(1+\delta_{\kappa_{0}} / 100 \%\right)=0.3286 \mathrm{MM}^{2} / \mathrm{c}$,

$a^{\max }=a \cdot\left(1+\delta_{a} / 100 \%\right)=0.6967 \mathrm{MM}^{2} / \mathrm{c}$,

$b^{\max }=b \cdot\left(1+\delta_{b} / 100 \%\right)=1.326$,

$\theta_{0}^{\max }=\theta_{0} \cdot\left(1+\delta_{\theta_{0}} / 100 \%\right)=0.070$

ле (5) они пропорциональны друг другу, поэтому, например, $\delta_{a} / \delta_{\kappa_{0}}=\alpha_{a} / \alpha_{\kappa_{0}}$. Если же говорить о количественных соотношениях, то в наших расчетах (при $\mathrm{CV}=20 \%$ ) $a$ вычисляется с относительной погрешностью в 4.4/0.41 $\approx 11$ раз

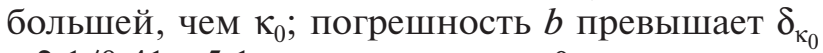
в $2.1 / 0.41 \approx 5.1$ раза и, наконец, $\theta_{0}$ определяется чрезвычайно плохо, ибо погрешность больше чем $\delta_{\kappa_{0}}$ в $9.3 / 0.41 \approx 23$ раза.

\section{Кратко о возможности регуляризации задачи идентификации}

Нетрудно заметить, что все рассмотренные выше примеры из [Архангельская, 2009], независимо от количества экспериментальных точек, которое различалось почти на порядок, обладают одной особенностью: задача идентификации $\theta_{0}$ оказывается плохо обусловленной (или, по крайней мере, близкой к таковой). Это вполне понятно. По физическому смыслу $\theta_{0}-$ это влажность, при которой достигается максимум температуропроводности, но форма графика зависимости $к(\theta)$ для всех этих примеров такова (монотонно возрастающая функция), что четкий максимум выделить невозможно см. рис. 1а. 
K настоящему времени разработана теория решения многих классов некорректных задач. Эти методы (методы регуляризации) [Амосов и др., 2008, с. 54], конечно же, могут с успехом применяться и для решения плохо обусловленных задач. Действительно, задача, некорректная в связи с тем, что не выполняется условие устойчивости по входным данным, представляет собой предельный случай плохо обусловленной задачи, а именно: $\alpha \rightarrow \infty$. Представляется вполне естественным, что метод, позволяющий решить задачу с бесконечно большим числом обусловленности, тем более сможет решить задачу, для которой $\alpha$ хотя и велико, но, все же, конечно.

Для решения некорректных задач существуют различные подходы - см., например, [Tikhonov and Arsenin, 1977; Амосов и др., 2008, с. 635-639, 644; Hansen, 2010; Glagolev and Sabrekov, 2019]. Но, в любом случае, возможность определения таких приближенных решений некорректной задачи, которые были бы устойчивы к малым изменениям исходных данных, основывается на использовании дополнительной информации относительно решения. Возможны различные типы этой дополнительной информации [Tikhonov and Arsenin, 1977]. Выше (в разд. «Результаты») мы, столкнувшись с плохой обусловленностью идентификации, дополнили задачу некоторой простейшей информацией - наложили априорные ограничения на искомые параметры. Эти ограничения были заданы из чисто логических соображений: $\kappa_{0}>0, a>0$ и $\theta_{\min }<\theta_{0}<\theta_{\max }$, где $\theta_{\min }=0, \theta_{\max }=1$. Но чем больше будет использовано информации, и чем она будет конкретнее, тем лучше станет обусловленность задачи.

Покажем это на примере задачи идентификации параметров зависимости температуропроводности от влажности: есть еще очевидная информация, которую мы пока не использовали для регуляризации решения. При увеличении $\theta$ объемное содержание воды в образце почвы увеличивается, а твердой и газообразной фаз - уменьшается. Очевидно, при $\theta=1$ мы имеем только воду. Следовательно, к(1) - это температуропроводность воды.

Согласно de Vries, теплопроводность воды при $10{ }^{\circ} \mathrm{C}$ составляет $1.37 \cdot 10^{-3}$ кал $/\left({ }^{\circ} \mathrm{C} \cdot \mathrm{cm} \cdot \mathrm{c}\right)$, а теплоемкость -1.00 кал $/\left({ }^{\circ} \mathrm{C} \cdot \mathrm{cm}^{3}\right)$ [Van Wijk, 1963 , Tab. 7.1]. Следовательно, $\kappa(1)=1.37 \cdot 10^{-7} \mathrm{M}^{2} / \mathrm{c}^{9}$. Таким образом, в (4) коэффициенты не могут быть произвольными, но должно выполняться равенство:

$$
0.137=\kappa_{0}+a \cdot \exp \left(-0.5 \cdot \ln ^{2}\left(1 / \theta_{0}\right) / b^{2}\right) .
$$

9 Воронин [1986, с. 223] (так же со ссылкой на de Vries), дает значение The $\mathrm{D}$ воды, равное $0.16 \cdot 10^{-3} \mathrm{M}^{2} / \mathrm{c}$. Такое значение не кажется нам реалистичным. (здесь мы выразили температуропроводность в $\mathrm{Mм}^{2} / \mathrm{c}$, поскольку с вычислительной точки зрения лучше, когда все параметры - примерно одного порядка). Из данного равенства можно найти любой коэффициент как функцию трех других, например,

$$
a=\left(0.137-\kappa_{0}\right) \cdot \exp \left(0.5 \cdot \ln ^{2}\left(1 / \theta_{0}\right) / b^{2}\right) .
$$

Поэтому (4) можно переписать в виде

$$
\begin{aligned}
\kappa=\kappa_{0}+ & \left(0.137-\kappa_{0}\right) \cdot \exp \left(0.5 \cdot \ln ^{2}\left(1 / \theta_{0}\right) / b^{2}\right) \times \\
& \times \exp \left(-0.5 \cdot \ln ^{2}\left(1 / \theta_{0}\right) / b^{2}\right) .
\end{aligned}
$$

Технически, во-первых, можно решать задачу идентификации параметров $\kappa_{0}, \theta_{0}$ и $b$ зависимости (8), после чего вычислить $a$ по формуле (7). A, во-вторых, можно использовать специальное программное обеспечение ${ }^{10}$, позволяющее минимизировать функцию - в данном случае это будет зависящая от $\kappa_{0}, \theta_{0}, b$ и $a$ невязка между экспериментальными данными и расчетом по формуле (4) - при заданном ограничении-равенстве - в данном случае таким ограничением явится формула (6) или любая другая, тождественная ей, например, формула (7).

Повторно решив задачу идентификации параметров (зависимости TheD от влажности) для горизонта $\mathrm{A}_{\text {пахII }}$ по данным [Архангельская, 2009, рис. 2б], но теперь уже используя не (4), а (8) и (6), т. е. принудительно заставив кривую пройти через точку $(1,0.137)$, мы получили очень хорошо обусловленную регуляризованную задачу во всем диапазоне CV $(1 \div 30 \%)$. Число обусловленности для $\kappa_{0}$ возрастало от 0.1 лишь до 0.6 , а для $b$, соответственно, от 0.3 до 0.7 (даже при $\mathrm{CV}=30 \%$ !); если же говорить о $\theta_{0}$ и $a$, то их числа обусловленности оставались на уровне $0.2 \div 0.3$ при любых CV.

Однако здесь есть другая опасность. Когда мы применяем регуляризацию, то любая используемая информация должна быть правильной. Функция (4) обладает определенными свойствами, которые задают достаточно жесткую связь, например, между величиной начального значения, положением и величиной максимума, и крутизной возрастания (или убывания) функции. Действительно, первые три перечисленные свойства определяются коэффициентами $\kappa_{0}, a$ и $\theta_{0}: \kappa(0)=\kappa_{0}, \max (\kappa)=\kappa_{0}+a$, $\arg \max (\kappa)=\theta_{0}$. Тогда подобрать крутизну, соответствующую экспериментальным данным,

${ }^{10}$ В качестве примеров таких программ можем указать MATLAB-функцию fmincon из Optimization Toolbox и целый ряд функций из библиотеки NAG: e04uca, e04ucf, e04ufa, e04uff, e04uga, e04ugf, e04vhf, e04wdf, причем специально для решения LeSP с ограничениями (в виде как равенств, так и неравенств) разработаны функции e04usa и e04usf. 
можно, варьируя $b$. При этом, если на самом деле функция (4) не соответствует физике процесса ${ }^{11}$, а является просто эмпирической зависимостью, то мы можем не заметить этого ведь за счет варьирования $b$ худо-бедно можно будет кривую в какой-то области «подогнать» к экспериментальным данным. И если экспериментальные данные покрывают лишь часть области определения к( $\theta)$, то никаких сложностей может не возникнуть. Если же (для обеспечения хорошей обусловленности) мы вводим какую-то дополнительную информацию, то в случае изначального выбора неправильной функции, не являющейся естественной для физики данного процесса, ее свойства могут прийти в противоречие с вводимой «физичной» информацией и какое-то из свойств мы потерям.

В частности, если мы вводим физически обоснованное условие (6), то на его обеспечение у нас уже нет свободных параметров - они все были задействованы для обеспечения соответствия других свойств функции экспериментальным данным ( $\kappa_{0}$ - величине начального значения, $a-$ величине максимума, $\theta_{0}-$ его положению, $b$ - крутизне). Поскольку в (6) входят все параметры, а они уже определены из других свойств функции, то требование выполнения (6) может привести к тому, что мы потеряем какое-то из ранее выполнявшихся свойств: или получим слишком большую или слишком малую величину начального или/и максимального значения, или/и положение максимума сместится, или/и крутизна кривой перестанет соответствовать экспериментальным данным.

В связи с этим плохо обусловленную задачу идентификации параметров таких зависимостей, как формула Архангельской, повидимому, нет смысла регуляризовывать, требуя точного соответствия каким-то строгим физическим соотношениям, поскольку эти соотношения могут войти в противоречие с эмпирической сутью данных зависимостей. Вероятно, в этом случае следует использовать более «мягкий» вариант, например - метод регуляризации Тихонова ${ }^{12}$ (подробнее о нем см., например, в [Tikhonov and Arsenin, 1977; Zhdanov, 2002; Амосов и др., 2008, с. 635-639, 644; Glagolev and Sabrekov, 2019]). Впрочем, для грамотного применения метода Тихонова критически важно знать уровень погрешности экспери-

${ }^{11}$ Т. е. информация о том, что формула (4) действительно задает температуропроводность при данной влажности, оказалась неправильной.

${ }^{12} \mathrm{~B}$ англоязычной литературе этот метод известен под названиями ("ridge regression", "weight decay", "Tikhonov-Miller method", "Tikhonov-Phillips regularization").

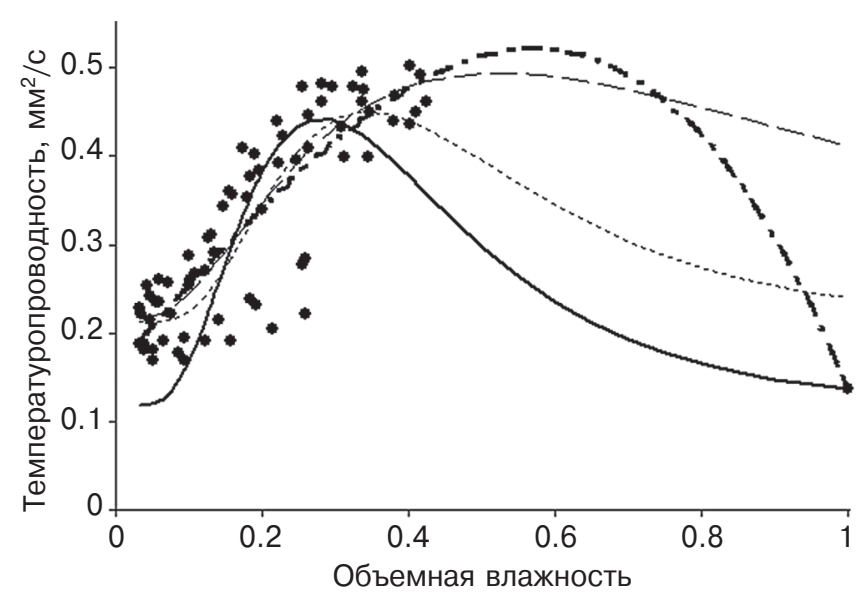

Pnc. 3. Аппроксимации зависимости температуропроводности горизонта $\mathrm{A}_{\text {пахII }}$ от влажности

Fis. 3. Approximations of the dependence of the thermal diffusivity of the horizon $\mathrm{A}_{\text {пахІІ }}$ from moisture

- - экспериментальные данные из [Архангельская, 2009];

- - - - - paсчет по (4) при идентификации параметров решением обычной LeSP $\left(\kappa_{0}=0.215 \mathrm{Mm}^{2} / \mathrm{c}\right.$, $a=0.279 \mathrm{mM}^{2} / \mathrm{c}, b=0.78, \theta_{0}=0.52$ [Архангельская, 2009, табл. 2]);

- расчет по (4) при идентификации параметров с «жесткой» регуляризацией равенством (6) $\left(\kappa_{0}=0.118 \mathrm{MM}^{2} / \mathrm{c}, a=0.323 \mathrm{MM}^{2} / \mathrm{c}, b=0.53, \theta_{0}=0.28\right) ;$

-......- - расчет по (4) при идентификации параметров одним из вариантов регуляризации Тихонова $\left(\kappa_{0}=0.212 \mathrm{MM}^{2} / \mathrm{c}, a=0.237 \mathrm{MM}^{2} / \mathrm{c}, b=0.51, \theta_{0}=0.35\right) ;$

...-.....- - полиномиальная аппроксимация $\kappa=-1.0232 \cdot \theta^{3}+0.07686 \cdot \theta^{2}+0.91898 \cdot \theta+0.16284$

ментальных данных, а здесь он, как уже было сказано, к сожалению не известен. Поэтому мы лишь проиллюстрируем на рис. 3 результаты, получаемые разными методами, но не будем претендовать на строгое решение задачи.

Для получения значений параметров, представленных на рис. 3, мы использовали следующий вариант метода регуляризации Тихонова: искали такие $\kappa_{0}, a, \theta_{0}$ и $b$, которые минимизируют функцию

$$
J\left(\kappa_{0}, a, \theta_{0}, b\right)=S+\Omega_{\alpha},
$$

здесь $S$ - сумма квадратов отклонений величин к, рассчитанных по (4), от соответствующих значений, полученных в эксперименте; $\Omega_{\alpha}-$ так называемый «регуляризатор», который в данном конкретном случае выбирался в виде $\Omega_{\alpha}=\alpha \cdot[\kappa(1)-0.137]^{2}$. Расчет, приведенный на рис. 3, получен при использовании значения $\alpha=2$ (но, вообще говоря, $\alpha$ должно определяться в соответствии с уровнем вариабельности экспериментальных данных). Если же говорить о формуле Архангельской, коэффициенты которой получены решением обычной LeSP (без ограничений - без регуляризации), то предсказываемое ею значение температуропроводности воды совершенно не соответствует действитель- 
ности, что говорит о сугубой эмпиричности этой формулы. Интересно отметить, что абсолютно эмпирическая аппроксимация полиномом ${ }^{13} 3$-го порядка дает весьма хороший результат, с одной стороны, описывая облако экспериментальных точек практически с тем же качеством, что и формула Архангельской, а, с другой, отлично удовлетворяя TheD воды при $\theta=1$.

Конечно, у специалиста в конкретной естественно-научной области (условного «физика») то или иное полученное решение может вызвать и обоснованные возражения. Например такое: слишком малые значения TheD (немногим более $0.1 \mathrm{mM}^{2} / \mathrm{c}$ ), которые получаются для низкой влажности при расчете с параметрами, определенными в результате «жесткой» регуляризации, никогда не наблюдались у сухих почв в реальных экспериментах или, может быть, считаются невозможными для горизонта $\mathrm{A}_{\text {пахII }}$ по каким-то другим почвоведческим соображениям. Эти возражсения необходимо учесть в математическом виде и вновь реиить задачу - с новым набором регуляризующих ограничений, включающим теперь и вышеуказанные возражсения (к сожалению, сразу учесть все необходимые ограничения, как правило, не удается).

Вообще, решая задачу, полезным является предъявление «физику» различных функций, каждая из которых может быть решением. Конкретные примеры возможных решений задачи часто содержат внешние дефекты, коих настоящее решение не должно иметь. В этом случае могут быть сформулированы дополнительные требования к решению, которые включаются в постановку задачи, решается новая, более сложная задача, и т. д. Опыт показывает, что такое извлечение априорной информации из «физика» успешнее проходит при предъявлении ему «решений», удовлетворяющих всем сформулированным им требованиям, но отвергаемых по каким-то интуитивным соображениям [Федоренко, 2008, с. 388]. По сути дела, для математика искусство работы с некорректными и плохо обусловленными прикладными задачами состоит в том, итобы «выведать» $y$ «физика» всю возможсную информацию, способную ограничить решение, и записать ее в виде тех или иных математических ограничений на решение.

${ }^{13}$ Мы специально рассмотрели и полином тоже, поскольку Архангельская [2005] в начале своей статьи показывает, что полиномиальная модель Тихонравовой-Хитрова на некоторых примерах работает хуже, чем (4), поэтому далее (в частности, в приложении к горизонту $\mathrm{A}_{\text {пахII }}$ ) она эту модель уже не рассматривает. Правда, указанная модель несколько отличается от полинома, рассмотренного нами.
Таким образом, задачу идентификации параметров зависимости температуропроводности от влажности (в случае ее плохой обусловленности) можно регуляризовать при помощи достаточно простых средств. Но теперь мы рассмотрим более общий и, можно сказать, «философский» вопрос: а нужно ли вообще определять коэффициент температуропроводности?

\section{Имеет ли температуропроводность смысл в задачах с переменными ThePOS?}

Цитированное выше (в разд. «Температуропроводность почвы») указание Воронина о том, что для расчета $T$ необходимо вынести $\lambda$ из-под знака производной, на наш взгляд - довольно странное. Впрочем, это заблуждение тогда было распространено довольно широко, затронув не только отечественных, но и зарубежных исследователей. В частности, Lettau [1971] тоже писал, что (2) может быть решено при заданных краевых условиях только если $C_{\mathrm{m}}$ и $\lambda$ не зависят, ни от $t$, ни от z. Но разве нельзя «определить изменения $T$ во времени на заданной глубине» просто решив уравнение (2)? Можно $^{14}$. Более того, не только можно, но нужно решать именно это уравнение, а не (3).

Предлагаемое Ворониным вынесение $\lambda$ за знак дифференциала допустимо лишь в том случае, когда зависимость $\lambda$ от $z$ столь слаба, что ею можно пренебречь и считать $\lambda$ постоянной величиной [Jury et al., 1991, p. 178]. Кстати, при получении уравнения (2) $\rho \cdot C_{\mathrm{m}}$ аналогичным образом выносится за знак дифференциала, поскольку изначально записывается уравнение (1) именно для изменения количества тепла (а не температуры!), и в это исходное уравнение, естественно, входит член $\partial\left(\rho \cdot C_{\mathrm{m}} \cdot T\right) / \partial t$, а не $\partial T / \partial t$.

Таким образом, необходимо обсудить наиболее существенный недостаток задачи (3), состоящий в предположении о постоянстве термических характеристик для почвы. Необходимость оценки степени этого допущения исключительно

14 Чтобы не быть голословными, приведем несколько ссылок на литературу, в которой описаны методы решения уравнения (2) или даже еще более общего случая - уравнения (1), причем переменные коэффициенты $C_{\mathrm{m}}$ и $\lambda$ могут зависеть не только от независимых переменных, но и от $T$ (при этом мы специально ограничимся лишь старыми книгами времен Lettau и Воронина). Численные методы решения будут рассмотрены далее (в разд. «Возникает ли коэффициент температуропроводности в разностной схеме?»), поэтому сейчас упомянем только приближенные аналитические методы. Их можно найти, например, в [Schechter, 1967; Коздоба, 1975; Farlow, 1982, Lecture 46]. 
важна и очевидна, ибо подобное предположение равносильно маловероятному утверждению о том, что почва является сплошным однородным твердым массивом. Учет изменчивости ThePOS приводит к более реальному формулированию задачи об отыскании температурного поля в почве [Нерпин и Чудновский, 1967, с. 239]. Но если, например, $\lambda$ не постоянна, то мы уже не можем просто вынести ее за знак дифференциала, а должны использовать правило дифференцирования произведения.

При этом, дифференцируя правую часть уравнения (2), получаем уравнение:

$$
\rho \cdot C_{\mathrm{m}} \cdot \partial T / \partial t=\partial \lambda / \partial z \cdot \partial T / \partial z+\lambda \cdot \partial^{2} T / \partial z^{2} .
$$

Если теперь обозначить $\partial \lambda / \partial z=\Lambda(z)$, то

$$
\partial T / \partial t=\left(\rho \cdot C_{\mathrm{m}}\right)^{-1} \cdot \Lambda(z) \cdot \partial T / \partial z+\kappa \cdot \partial^{2} T / \partial z^{2} .
$$

То, что более правильно записывать уравнение динамики температуры таким образом, а не в упрощенном виде (3), известно уже давно. По крайней мере около полувека назад такое уравнение опубликовал Lettau [1971, eq.(2)]. Тот же автор на конкретном примере расчета температурного поля сфагнового болота показал, что неучет члена $\left(\rho \cdot C_{\mathrm{m}}\right)^{-1} \cdot \Lambda(z) \cdot \partial T / \partial z$

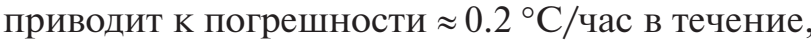
как минимум, 10 час. А это, следовательно, влечет за собой погрешность расчета температуры $\approx 2^{\circ} \mathrm{C}$. По его данным получается, что в течение суточного цикла в то время, когда температура почвы растет, оказывается, что $\kappa \cdot \partial^{2} T / \partial z^{2}>\left(\rho \cdot C_{\mathrm{m}}\right)^{-1} \cdot \Lambda(z) \cdot \partial T / \partial z$, но когда она охлаждается, $\kappa \cdot \partial^{2} T / \partial z^{2}<\left(\rho \cdot C_{\mathrm{m}}\right)^{-1} \cdot \Lambda(z) \cdot \partial T / \partial z$, причем лишь 25-33\% изменения температуры определяется тогда членом $\kappa \cdot \partial^{2} T / \partial z^{2}$.

Однако и в анализе Lettau [1971] принимается, что $\rho$ и $C_{\mathrm{m}}$ не изменяются с течением времени. Более правильным будет рассмотреть случай переменных $\rho$ и $C_{\mathrm{m}}$. Непостоянство этих величин может иметь место, например, тогда, когда изменяется влажность почвы.

Количество влаги в почве очень изменчиво во времени и зависит от соотношения поступления воды в почву (с атмосферными осадками, поливными и грунтовыми водами) и расходования ее из почвы (испарение, транспирация, сток и т. д.) [Растворова, 1983, с. 54]. Температура и осадки, определяющие это в первую очередь, характеризуются относительно быстрыми и сильными изменениями [Клюкин, 1960; Лебедев, 1964; Звягина и Переясловец, 2018; Таланова, 2018]. Влияние же влажности и плотности на теплофизические свойства грунтов исключительно велико. Именно колебания этих величин определяют широкий диапазон ThePOS в сезонно-талом или промерзающем слоях. Например, при увлажнении грунтов от воздушно сухого состояния до полного влагонасыщения коэффициент теплопроводности может возрасти в 8-10 раз [Гаврильев, 2004, с. 16]. Более того, Г.Н. Федотов и Д.В. Жуков [2004] выявили достаточно сложную кинетику процесса: показав, что при внесении воды в почвы, высушенные до воздушно или абсолютно сухого состояний, TheD уменьшаются в течение первых 3 суток после добавления воды в сухие образцы, однако в дальнейшем начинают расти ${ }^{15}$. Не обойдены вниманием исследователей и другие факторы среды.

В частности, получены зависимости коэффициента температуропроводности от температуры во-первых, для почв, в которых органоминеральные гелевые структуры были разрушены (сразу после добавления воды) и, во-вторых, когда они уже восстановились. В последнем случае с ростом температуры наблюдается сначала рост TheD, а затем спад до значений, меньших исходных [Федотов и Жуков, 2004]. Зависимость коэффициента температуропроводности от температуры для целого ряда почв (песчаных, суглинистых, торфяных) исследовалась в работах известного специалиста - Ларисы Эдуардовны Лапиной [Лапина, 2019; Лапина и Воропай, 2019].

В свете этого, дифференцируя в левой части (1) не просто $T$, а $\left(\rho \cdot C_{\mathrm{m}} \cdot T\right)$, получаем:

$$
\begin{gathered}
T \cdot \partial\left(\rho \cdot C_{\mathrm{m}}\right) / \partial t+\rho \cdot C_{\mathrm{m}} \cdot \partial T / \partial t= \\
\quad=\partial \lambda / \partial z \cdot \partial T / \partial z+\lambda \cdot \partial^{2} T / \partial z^{2} .
\end{gathered}
$$

Если теперь обозначить $\partial\left(\rho \cdot C_{\mathrm{m}}\right) / \partial t=\mathrm{B}(t)$, то

$$
\begin{gathered}
\left(\rho \cdot C_{\mathrm{m}}\right)^{-1} \cdot T \cdot \mathrm{B}(t)+\partial T / \partial t= \\
=\left(\rho \cdot C_{\mathrm{m}}\right)^{-1} \cdot \Lambda(z) \cdot \partial T / \partial z+\kappa \cdot \partial^{2} T / \partial z^{2} .
\end{gathered}
$$

Итак, для понимания явлений, связанных с термическим режимом почвы, требуется знание комплекса величин, именуемых термическими $^{16}$ характеристиками почвы; в этот комплекс входят коэффициенты тепло- и температуропроводности, а также теплоемкость [Нерпин и Чудновский, 1967, с. 238]. Мы особо отмечаем это, поскольку, на наш взгляд, ряд специалистов, уповая на простейшее уравнение (3), из всех ThePOS определяют одну лишь к. Но подчеркнем, что необходимость использования других ThePOS возникает при условии их непостоянства. В результате чего это непостоянство может проявиться? Очевидно,

${ }^{15}$ Отметим, что это не единичное наблюдение. Данный эффект был обнаружен для всего широкого спектра исследованных Федотовым и Жуковым [2004] почв: кубанского выщелоченного чернозема, торфяной и дерново-подзолистой из поймы р. Яхрома и ее окрестностей, а также тепличного субстрата.

${ }^{16}$ А.Ф. Чудновский $[1976$, с. 8] называет их не «термическими», а «теплофизическими». 
Таблица / Table

Уравнения Аинамики температурного поля почвы.

Equations of dynamics of the soil temperature field.

\begin{tabular}{|c|c|c|c|}
\hline \multirow{2}{*}{$\begin{array}{c}\text { Объемная } \\
\text { теп^оемкость } \\
\left(\mathbf{C}=\rho \cdot \mathbf{C}_{\mathrm{m}}\right)\end{array}$} & \multicolumn{3}{|c|}{ ТеплопровоАность $(\lambda)$} \\
\hline & постоянна: $\lambda=$ const & зависит от времени: $\lambda=\lambda(t)$ & зависит от глубины: $\lambda=\lambda(z)$ \\
\hline$C=$ const & $\partial T / \partial t=\kappa \cdot \partial^{2} T / \partial z^{2}$ & $\partial T / \partial t=\kappa(t) \cdot \partial^{2} T / \partial z^{2}$ & $\partial T / \partial t=\partial[\kappa(z) \cdot \partial T / \partial z] / \partial z$ \\
\hline$C=C(t)$ & $\partial\left[\kappa(t)^{-1} \cdot T / \partial t\right]=\partial^{2} T / \partial z^{2}$ & $\begin{array}{l}C(t)^{-1} \cdot T \cdot \partial C / \partial t+\partial T / \partial t= \\
=\kappa(t) \cdot \partial^{2} T / \partial z^{2}\end{array}$ & $\begin{aligned} C(t)^{-1} \cdot T \cdot \partial C / \partial t & +\partial T / \partial t= \\
& =\partial[\kappa(z) \cdot \partial T / \partial z] / \partial z\end{aligned}$ \\
\hline$C=C(z)$ & $\partial T / \partial t=\kappa(z) \cdot \partial^{2} T / \partial z^{2}$ & $\partial T / \partial t=\kappa(z, t) \cdot \partial^{2} T / \partial z^{2}$ & $\begin{aligned} \partial T / \partial t=C(z)^{-1} \cdot \partial \lambda / \partial z & \cdot \partial T / \partial z+ \\
& +\kappa(z) \cdot \partial^{2} T / \partial z^{2}\end{aligned}$ \\
\hline
\end{tabular}

что в результате изменения факторов (параметров) внешней среды, как со временем, так и в пространстве - по почвенному профилю.

В вышеприведенной таблице суммированы возможные комбинации зависимостей теплоемкости и теплопроводности от времени и глубины. Из табл. видно, что если $\rho \cdot C_{m}=$ const, то в уравнении динамики температурного поля коэффициент температуропроводности появляется вполне естественным образом, впрочем, мы это видели выше при выводе уравнения (3). Одного этого коэффициента оказывается достаточно (никакие другие ThePOS не нужны) для расчета динамики температуры почвы; но, разумеется, если коэффициент теплопроводности зависит от времени, то и TheD зависит от времени (аналогично - в случае зависимости от глубины). Также одного лишь коэффициента температуропроводности оказывается достаточно и при $\lambda=$ const (при этом зависимость теплоемкости от $t$ или $z$ порождает зависимость The D, соответственно, от $t$ или $z$ ). Последняя ситуация, когда достаточно одного лишь коэффициента температуропроводности - это случай зависимости теплоемкости только от $z$, а теплопроводности - только от $\boldsymbol{t}$. Поскольку при этом дифференцируемые коэффициенты зависят не от тех переменных, по которым берутся частные производные, то они могут быть вынесены за знаки производных, но, очевидно, TheD будет в этом случае зависеть от обеих независимых переменных: $\kappa(z, t)=\lambda(t) / \mathrm{C}(z)$. В остальных случаях (т. е. когда $C$ зависит от $t$, а $\lambda-$ от $z$; или когда оба коэффициента - и $C$, и $\lambda-$ зависят от одной и той же независимой переменной: либо оба от $t$, либо оба от $z$ ) в уравнении динамики температуры неизбежно появляются два коэффициента, т. е. ограничиться одной лишь температуропроводностью невозможно. Для практических расчетов, безусловно, важен вопрос: какие случаи, из числа тех, что рассмотрены в табл., наиболее реалистичны?

Очевидно, что в неоднородном верхнем слое почвы не выполняется условие постоянства $\lambda$ [Lettau, 1971] по пространству (по глубине).
Это сразу исключает два первых столбца таблицы из числа претендентов на звание «реалистичных случаев». Но постоянство $C$ (первая строка таблицы) тоже приходится исключить, поскольку хорошо известно, что теплоемкость почвы зависит от влажности (см., например, [Воронин, 1986, с. 223-224; Jury et al., 1991, p. 179; Гаврильев, 2004, с. 14; Шеин, 2005, с. 328]), а влажность, в свою очередь, изменяется с глубиной в большинстве почв [Нерпин и Чудновский, 1967, с. 206; Ревут, 1972, с. 283; Растворова, 1983, с. 100-102; Mochenov et al., 2018]. Следовательно, реалистичными могут быть лишь два случая: $C=C(t), \lambda=\lambda(z)$ и $C=C(z), \lambda=\lambda(z)$, т. е. именно те, когда для расчета динамики температурного поля невозможно ограничиться одной лишь температуропроводностью.

\section{Возникает ли коэффициент темпера- туропроводности в разностной схеме?}

Иногда «специалисты», занимающиеся толь$\boldsymbol{\kappa} \boldsymbol{о}$ измерением температуропроводности, на вопрос о том, зачем же это нужно, отвечают ${ }^{17}$, что ныне все задачи решаются численно, а при этом почвенный профиль разбивается на отдельные слои, каждому из которых приписывается свое постоянное значение к. Разберем, так ли это.

Вообще говоря, для решения уравнения (2) предложено множество методов (см., например, [Самарский, 1983; Shih, 1984; Федоренко, 2008, с. 102-130; Шеин и Рыжова, 2016, с. 208-211]) и создано огромное количество реализующих их компьютерных программ (описания некоторых из них см. в [Бартеньев, 2001; Глаголев и Смагин, 2005, с. 90-111; Коваленко и др., 2017; Глаголев и др., 2019]). Поэтому дать общий ответ, вероятно, нельзя. Но можно рассмотреть часто используемые методы и убедиться, что, по крайней мере для них это не так.

${ }^{17}$ В частности, так ответила на соответствующий вопрос авторов одна из докладчиков на 6-ой Национальной научной конференции с международным участием «Математическое моделирование в экологии» (Пущино, 2019 г.). 
Shih [1984, Table 6-8] дает следующую конечно-разностную аппроксимацию для члена кондуктивного теплопереноса на $i$-ом пространственном «слое» конечно-разностной сетки (ПроСКРаС):

$$
\begin{aligned}
& \partial(\lambda \cdot \partial T / \partial z) / \partial z \approx\left(0.5 / h^{2}\right) \cdot\left[\left(\lambda_{i}+\lambda_{i+1}\right) \cdot T_{i+1}-\right. \\
& \left.-\left(\lambda_{i-1}+2 \cdot \lambda_{i}+\lambda_{i+1}\right) \cdot T_{i}-\left(\lambda_{i-1}+\lambda_{i}\right) \cdot T_{i-1}\right],
\end{aligned}
$$

где $\lambda_{i-1}, \lambda_{i}, \lambda_{i+1}$ и $T_{i-1}, T_{i}, T_{i+1}-$ соответственно, значения $\lambda$ и $T$ на $(i-1)$-ом, $i$-ом и $(i+1)$-ом ПроСКРаС, т. е. $\lambda_{i-1}=\lambda\left(z_{i-1}\right), \lambda_{i}=\lambda\left(z_{i}\right)$ и т. д.; $h$ - шаг по оси $z$ (т. е. $h=z_{i}-z_{i-1}=z_{i+1}-z_{i}$ ). На каком бы ПроСКРаС ни аппроксимировалась левая часть уравнения (2), мы не сможем получить в результирующем конечно-разностном уравнении только температуропроводность. Действительно, простейшей является аппроксимация левой части уравнения (2) просто на $i$-ом слое. Но тогда

$$
\begin{gathered}
C_{i} \cdot A\left(T_{i}\right) \approx\left(0.5 / h^{2}\right) \cdot\left[\left(\lambda_{i}+\lambda_{i+1}\right) \cdot T_{i+1}-\right. \\
\left.-\left(\lambda_{i-1}+2 \cdot \lambda_{i}+\lambda_{i+1}\right) \cdot T_{i}-\left(\lambda_{i-1}+\lambda_{i}\right) \cdot T_{i-1}\right] .
\end{gathered}
$$

Здесь через $C_{i}$ и $A\left(T_{i}\right)$ мы обозначили, соответственно, значения на $i$-ом слое произведения $\rho \cdot C_{\mathrm{m}}$ и конечно-разностной аппроксимации производной $T$ по $t$. Деля полученное уравнение на $C_{i}$, имеем:

$$
\begin{gathered}
A\left(T_{i}\right) \approx\left(0.5 / h^{2}\right) \cdot\left[\left(\kappa_{i}+\lambda_{i+1} / C_{i}\right) \cdot T_{i+1}-\right. \\
-\left(\lambda_{i-1} / C_{i}+2 \cdot \kappa_{i}+\lambda_{i+1} / C_{i}\right) \cdot T_{i}- \\
\left.-\left(\lambda_{i-1} / C_{i}+\kappa_{i}\right) \cdot T_{i-1}\right],
\end{gathered}
$$

где $\kappa_{i}-$ значение TheD на $i$-ом ПроСКРаC, но $\lambda_{i+1} / C_{i}$ и $\lambda_{i-1} / C_{i}$ какими-либо коэффициентами температуропроводности не являются, поскольку в этих отношениях значения числителя и знаменателя берутся с разных слоев (т. е., в частности, при разных значениях влажности), тогда как в TheD они должны соответствовать одному и тому же слою (одному и тому же значению влажности, температуры, текстуры и др.). Аналогичную ситуацию имеем и в других разностных схемах.

Вообще говоря, при написании разностных схем следует добиваться, чтобы эти схемы выражали на сетке соответствующий закон сохранения. Такие схемы называются консервативными [Самарский, 1983, с. 143]. В случае теплопереноса они выражают закон сохранения энергии (тепла). И именно такие схемы применяются при моделировании динамики температурного поля почв и грунтов в серьезных исследованиях (см., например, [Попов, 1995, с. 25; Мордовской и др., 1997, с. 33; Калюжный и Лавров, 2012]).

Для уравнения (2) консервативная разностная схема аппроксимирует член кондуктивного теплопереноса на $i$-ом ПроСКРаС следующим образом:

$$
\begin{gathered}
\partial(\lambda \cdot \partial T / \partial z) / \partial z \approx \\
\approx\left[\left(T_{i+1}-T_{i}\right) \cdot a_{i+1}-\left(T_{i}-T_{i-1}\right) \cdot a_{i}\right] / h^{2}, \\
\text { где } a_{i}=\left[\frac{1}{h} \int_{z_{i-1}}^{z_{i}} \frac{d z}{\lambda(z)}\right]^{-1} .
\end{gathered}
$$

Но для практических целей удобно иметь возможно более простые формулы нахождения $a_{i}$, использующие значения $\lambda$ в отдельных точках. Обычно рассматривают шаблон из одной или двух точек, полагая, например,

$$
\begin{gathered}
a_{i}=\lambda_{i-1 / 2}, \\
a_{i}=0.5 \cdot\left(\lambda_{i}+\lambda_{i-1}\right)
\end{gathered}
$$

или $a_{i}=2 \cdot \lambda_{i} \cdot \lambda_{i-1} /\left(\lambda_{i}+\lambda_{i-1}\right)$ [Самарский, 1983, c. 145,148$]$. В частности, Ф.С. Попов [1995, с. 25] и С.Д. Мордовской и др. [1997, с. 33] используют (13) ${ }^{18}$, а Калюжный и Лавров [2012] рекомендуют (14), считая эту формулу простейшей.

Сама по себе аппроксимация (12), независимо от используемой формулы для $a_{i}$, уже приводит к тому, что одним лишь коэффициентом температуропроводности обойтись не удается. Действительно, используя (12), имеем вместо (11) конечно-разностную схему вида

$$
\begin{gathered}
A\left(T_{i}\right) \approx \\
\approx\left[\left(T_{i+1}-T_{i}\right) \cdot a_{i+1} / C_{i}-\left(T_{i}-T_{i-1}\right) \cdot a_{i} / C_{i}\right] / h^{2},
\end{gathered}
$$

следовательно, какую бы формулу для $a_{i}$ мы ни использовали, член $a_{i+1} / C_{i}$ невозможно будет выразить через TheD. Более того, если применять (13), то в разностной схеме коэффициент температуропроводности вообще не появится! В самом деле, в этом случае схема примет вид

$$
\begin{gathered}
A\left(T_{i}\right) \approx \\
\approx\left[\left(T_{i+1}-T_{i}\right) \cdot \lambda_{i+1 / 2} / C_{i}-\left(T_{i}-T_{i-1}\right) \cdot \lambda_{i-1 / 2} / C_{i}\right] / h^{2} .
\end{gathered}
$$

Ни $\lambda_{i+1 / 2} / C_{i}$, ни $\lambda_{i-1 / 2} / C_{i}$ каким-либо коэффициентом температуропроводности не являются, поскольку в этих отношениях значения числителя и знаменателя берутся с разных слоев $^{19}$ (т. е., в частности, при разных значениях влажности).

18 Поскольку уравнение (2) весьма похоже на уравнение Ричардса (движения влаги в почве), то последнее решается по аналогичным разностным схемам, и, например, в учебнике [Шеин и Рыжова, 2016 , с. 209] совершенно справедливо используется формула (13) для решения уравнения Ричардса (хотя авторы и не акцентируют внимание студентов на том, что схема является консервативной).

${ }^{19}$ Если использовать терминологию теории разностных схем, то, строго говоря, $C_{i}$ берется на $i$-ом слое, а $\lambda_{i+1 / 2}$ и $\lambda_{i-1 / 2}-$ на последующем и предыдущем полуслоях. 
В свете сказанного выше становится очевидным почему в цитированных выше работах [Попов, 1995; Мордовской и др., 1997; Калюжный и Лавров, 2012], как и во многих других, посвященных серьезному моделированию сложных задач динамики температуры в реальных природных системах, никаких коэффициентов температуропроводности вообще не возникает (по крайней мере, в тех разделах, где решается уравнение теплопереноса). Авторы исследуют зависимости теплоемкости и теплопроводности от факторов среды (например, от той же влажности) и далее работают с уравнением (15), выражая из него $T_{i}-$ искомую расчетную температуру $i$-го слоя.

\section{BblBOabl}

1) Задачи идентификации параметров зависимости коэффициента температуропроводности от влажности часто оказываются плохо обусловленными.
2) Их обусловленность может быть улучшена с помощью регуляризующих алгоритмов, основывающихся на использовании дополнительной информации о решении.

3) Однако в наиболее реалистичных случаях (когда объемная теплоемкость изменяется во времени или в пространстве, а теплопроводность - в пространстве) одного лишь коэффициента температуропроводности оказывается недостаточно для моделирования динамики температурного поля.

4) Эта ситуация не зависит от того, используются ли для расчета аналитические или численные методы.

\section{БПАГОЯАРНОСТЬ}

Авторы искренне благодарят двух анонимных рецензентов за содержательный разбор статьи и множество полезных советов, некоторые из которых были отмечены выше в подстраничных сносках. Работа выполнена по теме госзадания №AAAA-A15-115122810146-4.

\section{ПРИЛОЖЕНИЕ: ТЕКСТ МАТLАВ-ПРОГРАММЫ}

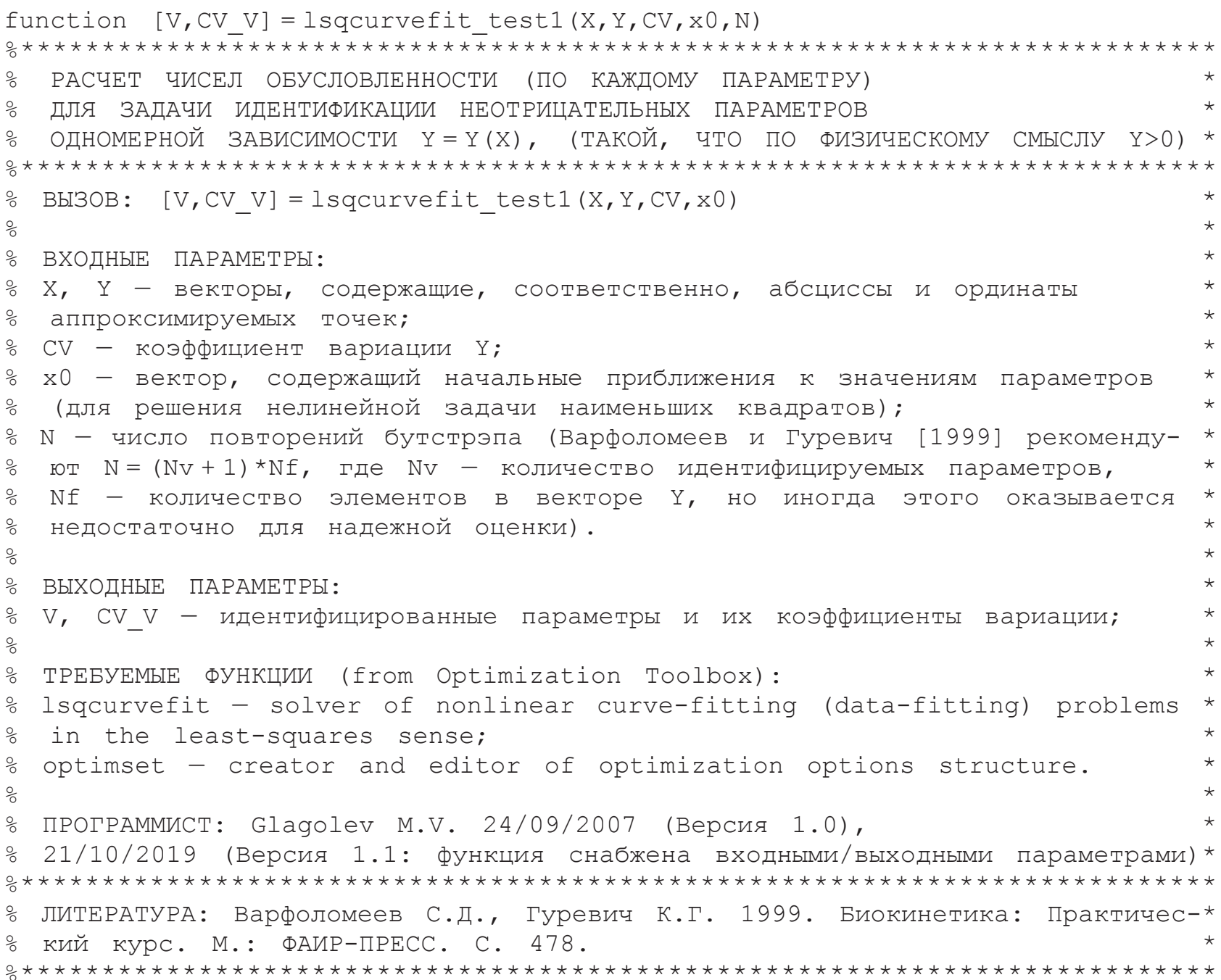




\section{Окончание ПРИЛОЖЕНИЕ: ТЕКСТ МАТLАВ-ПРОГРАММЫ}

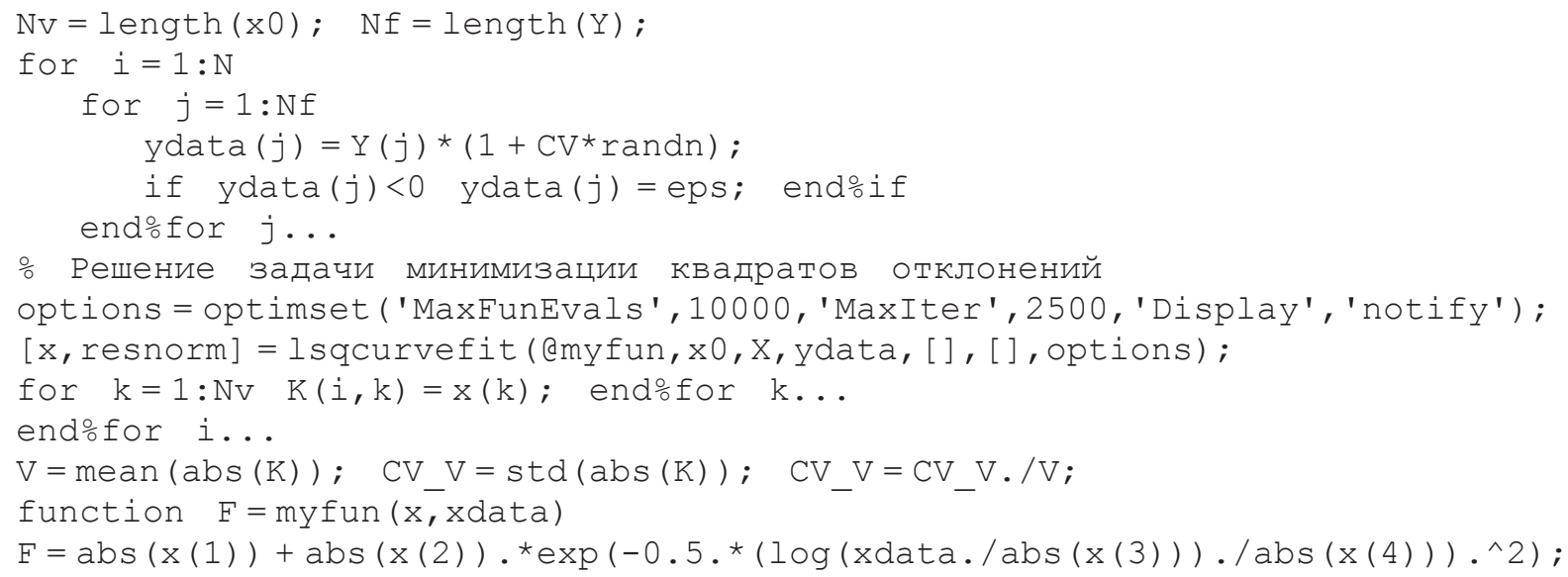

\section{กИTEPATYPA}

1. Амосов АА, Дубинский ЮА, Копченова НВ, 2008. Вычислительные методы. Издат. дом МЭИ, Москва: 672 с. [Amosov AA, Dubinskiy YuA, Kopchenova NV, 2008. Vychislitel'nye Metody. Izdat. dom MEI, Moscow: 672 pp. (In Russian)].

2. Архангельская ТА, 2004. Новая эмпирическая фрормула для оценки коэффициента температуропроводности почвы, с. 45-46 В: Материалы научной сессии по фундаментальному почвоведению, Москва [Arkhangel'skaya TA, 2004. Novaya empiricheskaya formula dlya otsenki koeffitsienta temperaturoprovodnosti pochvy, p. 45-46 In: Materialy nauchnoy sessii po fundamental'nomu pochvovedeniyu, Moscow (In Russian)].

3. Архангельская ТА, 2009. Параметризация и математическое моделирование зависимости температуропроводности почвы от влажности. Почвоведение. 2:178-188. [Arkhangel'skaya TA, 2009. Parameterization and mathematical modeling of the dependence of soil thermal diffusivity on the water content. Eurasian Soil Science. 42:162-172].

4. Бартеньев OB, 2001. Фортран для профессионалов. Математическая библиотека IMSL. 4. 3. Диалог-МИФИ, Москва: 320 с. [Barten'ev OV, 2001. Fortran Dlya Professionalov. Matematicheskaya Biblioteka IMSL. P. 3. Dialog-MIFI, Moscow: 320 pp. (In Russian)].

5. Варфоломеев СД, Гуревич КГ, 1999. Биокинетика: Практический курс. ФАИР-ПРЕСС, Москва: 720 с. [Varfolomeev SD, Gurevich KG, 1999. Biokinetika: Prakticheskiy Kurs. FAIR-PRESS, Moscow: 720 pp. (In Russian)].

6. Воронин АД, 1986. Основы фризики почв. Изд-во МГУ, Москва: 244 с. [Voronin AD, 1986. Osnovy fiziki pochv. Izd-vo MGU, Moscow: 244 pp. (In Russian)].

7. Гаврильев РИ, 2004. Теплофизические свойства компонентов природной среды в криолитозоне. Изд-во СО РАН, Новосибирск: 146 с. [Gavril'ev RI, 2004. Teplofizicheskie Svoystva Komponentov Prirodnoy Sredy v Kriolitozone. Izdvo SO RAN, Novosibirsk: 146 pp. (In Russian)].

8. Глаголев MB, Сабреков АФ, 2019. Плохо обусловленные математические задачи ффизики почв, с. 271-275 B: Фундаментальные концепции фризики почв: развитие, современные приложения и перспективы: Сборник науч- ных трудов Международной научной конференции, посвященной 90-летию со дня рождения Анатолия Даниловича Воронина. Москва [Glagolev MV, Sabrekov AF, 2019. III-conditioned mathematical problems of soil physics, p. 271-275 In: Proceedings of the International scientific conference, organized to commemorate the 90th anniversary of A.D. Voronin's birth "Key concepts of soil physics: development, future prospects and current applications" Moscow (In Russian, English abstract)].

9. Глаголев МВ, Сабреков АФ, Казанцев ВС, 2010. Физикохимия и биология торфа. Методы измерения газообмена на границе почва-атмосфера. Изд-во ТГПУ, Томск: 104 с. [Glagolev MV, Sabrekov AF, Kazantsev V, 2010. Fizikokhimiya i Biologiya Torfa. Metody Izmereniya Gazoobmena Na Granitse Pochva-Atmosfera. Izd-vo TGPU, Tomsk: 104 pp. (In Russian)].

10. Глаголев MB, Смагин AB, 2005. Приложения MATLAB для численных задач биологии, экологии и почвоведения. МГУ им. М.В. Ломоносова, Москва: 200 с. [Glagolev MV, Smagin AV, 2005. Matlab Applications for Numerical Simulations in Biology, Ecology and Soil Science. MGU im. M.V. Lomonosova, Moscow: 200 pp. (In Russian)].

11. Глаголев MB, Фаустова EB, Сабреков АФ, Гончаров ВМ, 2019. Численное решение уравнений биокинетики в курсах «Общая экология» и «Моделирование биологических процессов». Том II. Уравнения в частных производных. КДУ, Университетская книга, Москва: 220 с. [Glagolev MV, Faustova EV, Sabrekov AF, Goncharov VM, 2019. Chislennoe Reshenie Uravneniy Biokinetiki v Kursakh "Obshchaya Ekologiya" i "Modelirovanie Biologicheskikh Protsessov". II. Uravneniya v Chastnykh Proizvodnykh. KDU, Universitetskaya kniga, Moscow: 220 pp. (In Russian)].

12. Еремеев НЛ, Карякин АА, Казанская НФ, 1989. Кинетика растворения твердых белковых субстратов протеиназами. Выбор механизма реакции. Биохимия 54:503-510. [Eremeev NL, Karyakin AA, Kazanskaya NF, 1989. Kinetics of dissolution of solid protein substrates by proteinases. Selection of the reaction mechanism Biochemistry (USSR). 54:503-510 (In Russian, English Abstract)].

13. Звягина ЕА, Переясловец ТС, 2018. Динамика метеорологических показателей и продолжительности вегетаци- 
онного периода в Юганском заповеднике 1961-2016 гг. Динамика окружающей среды и глобальные изменения климата. 9:28-39. [Zvyagina EA, Pereyaslovets TS, 2018. Long-term phenoclimatic averages of the Yuganskiy Nature Reserve territory and changes for 1961-2016. Environmental dynamics and global climate change 9:28-39 (In Russian, English Abstract)]. doi: 10.17816/edgcc10367

14. Зубарев ДН, 1998. Термодифффузия. Физическая энциклопедия. [Zubarev DN, 1998. Termodiffuziya. Fizicheskaya entsiklopediya. (In Russian)].

15. Калюжный ИЛ, Лавров СА, 2012. Гидрофизические процессы на водосборе. Экспериментальные исследования и моделирование. Нестор-История, Санкт-Петербург: 156161 c. [Kalyuzhnyy IL, Lavrov SA, 2012. Gidrofizicheskie protsessy na vodosbore. Eksperimental'nye issledovaniya i modelirovanie. Nestor-Istoriya, Saint-Petersburg: 156161 pp. (In Russian)].

16. Клюкин НК, ред, 1960. Прикладной климатологический справочник Северо-Востока СССР. Альбом карт. Магаданское кн. изд-во, Магадан: 25 с. [Klyukin NK, editor, 1960. Prikladnoy klimatologicheskiy spravochnik severovostoka SSSR. Al'bom Kart. Magadanskoe kn. izd-vo., Magadan: 25 pp. (In Russian)].

17. Коваленко АВ, Узденова АМ, Уртенов МX, Никоненко ВB, 2017. Математическое моделирование физико-химических процессов в среде Comsol Multiphysics 5.2. Изд-во Лань, СПб.: 228 с. [Kovalenko AV, Uzdenova AM, Urtenov MKh, Nikonenko VV, 2017. Matematicheskoe modelirovanie fiziko-khimicheskikh protsessov v srede comsol multiphysics 5.2. Izd-vo Lan', Saint-Petersburg: 228 pp. (In Russian)].

18. Коздоба ЛА, 1975. Методы решения нелинейных задач теплопроводности. Наука, Москва: 228 с. [Kozdoba LA, 1975. Metody resheniya nelineynykh zadach teploprovodnosti. Nauka, Moscow: 228 pp. (In Russian)].

19. Котоусов ЛС, 1973. Термодиффузия - метод исследования неидеальных систем. Наука, Ленинград: 199 с. [Коtousov LS, 1973. Termodiffuziya — Metod Issledovaniya Neideal'nykh Sistem. Nauka, Leningrad: 199 pp. (In Russian)].

20. Куртенер ДА, Чудновский АФ, 1979. Агрометеорологические основы тепловой мелиорации почв. Гидрометеоиздат, Ленинград: 232 с. [Kurtener DA, Chudnovskiy AF, 1979. Agrometeorologicheskie Osnovy Teplovoy Melioratsii Pochv. Gidrometeoizdat, Leningrad: 232 pp. (In Russian)].

21. Лапина ЛЭ, 2019. Зависимость коэффициента температуропроводности от температуры среды, с. 748-751 В: Фундаментальные концепции фризики почв: развитие, современные приложения и перспективы: Сборник научных трудов Международной научной конференции, посвященной 90-летию со дня рождения Анатолия Даниловича Воронина. Москва. [Lapina LE, 2019. The dependence of the thermal diffusivity of the soil temperature, p. 748-751 In: Proceedings of the International scientific conference, organized to commemorate the 90th anniversary of A.D. Voronin's birth "Key concepts of soil physics: development, future prospects and current applications," Moscow (In Russian)].

22. Лапина ЛЭ, Воропай НН, 2019. Зависимость функции температуропроводности от температуры почвы на при- мере песчаных отложений массива Бадар (Тункинская котловина), с. 198-199 В: XIII-ое Сибирское совещание и школа молодых ученых по климато-экологическому мониторингу, Томск. [Lapina LE, Voropay NN, 2019. Zavisimost' funktsii temperaturoprovodnosti ot temperatury pochvy na primere peschanykh otlozheniy massiva Badar (Tunkinskaya kotlovina), p. 198-199 In: XIII-oe Sibirskoe soveshchanie i shkola molodykh uchenykh po klimato-ekologicheskomu monitoringu, Tomsk (In Russian, English Abstract)].

23. Лебедев АА, Архангельская ТА, Початкова ТН, Тюгай 3Н, 2019. Температуропроводность серогумусовых песчаных почв, с. 757-762. В: Фундаментальные концепции фризики почв: развитие, современные приложения и перспективы: Сборник научных трудов Международной научной конференции, посвященной 90-летию со дня рождения Анатолия Даниловича Воронина. Москва. [Lebedev AA, Arkhangelskaya TA, Pochatkova TN, Tyugai ZN, 2019. Thermal diffusivity of lammelic arenosols as related to soil moisture p. 757-762 In: Proceedings of the International scientific conference, organized to commemorate the 90th anniversary of A.D. Voronin's birth "Key concepts of soil physics: development, future prospects and current applications," Moscow (In Russian, English Abstract)].

24. Лебедев АН, 1964. Продолжительность дождей на территории СССР. Гидрометеоиздат: Ленинград: 223 c. [Lebe$\operatorname{dev}$ AN, 1964. Prodolzhitel'nost' Dozhdey Na Territorii SSSR. Gidrometeoizdat, Leningrad: 223 pp. (In Russian)].

25. Мордовской СД, Петров ЕЕ, Изаксон ВЮ, 1997. Математическое моделирование двухфазной зоны при промерзании-протаивании многолетнемерзлых пород. Наука, Новосибирск: 120 с. [Mordovskoy SD, Petrov EE, Izakson VYu, 1997. Matematicheskoe Modelirovanie Dvukhfaznoy Zony Pri Promerzanii-Protaivanii Mnogoletnemerzlykh Porod. Nauka, Novosibirsk: 120 pp. (In Russian)].

26. Моченов СЮ, Чуркина АИ, Глаголев МВ, 2017. Анализ корректности почвенно-градиентного метода измерения эмиссии газов, с. 131-133 В: Математическое моделирование в экологии, Пущино. [Mochenov SYu, Churkina Al, Glagolev MV, Analiz korrektnosti pochvenno-gradientnogo metoda izmereniya emissii gazov, p. 131-133 In: Matematicheskoe modelirovanie v ekologii, Pushchino (In Russian)].

27. Набиев ЭЮ, Гусейнов СБ, 1990. О зависимости коэффициента температуропроводности почв от содержания фризической глины. Почвоведение. 10:149-151. [Nabiev EYu, Guseynov SB, 1990. 0 zavisimosti koeffitsienta temperaturoprovodnosti pochv ot soderzhaniya fizicheskoy gliny. Soviet Soil Science 10:149-151 (In Russian)].

28. Нерпин СВ, Чудновский АФ, 1967. Физика почвы. Наука, Москва: 584 с. [Nerpin SV, Chudnovskiy AF, 1967. Fizika Pochvy. Nauka, Moscow: 584 pp. (In Russian)].

29. Орлов ДС, Минько ОИ, Аммосова ЯМ, Каспаров СВ, Глаголев МВ, 1987. Методы исследования газовой функции почвы, с. 118-156 В: А.Д. Воронин и Д.С. Орлов (ред.). Современные физические и химические методы исследования почв, Москва: Изд-во МГУ. [Orlov DS, Min'ko Ol, Ammosova YaM, Kasparov SV, Glagolev MV, 1987. Metody issledovaniya gazovoi funktsii pochvy, p. 118-156 In: A.D. Voronin and D.S. Orlov (eds.), Sovremennye fizicheskie 
i khimicheskie metody issledovaniya pochv, Moscow: Izd-vo MGU (In Russian)].

30. Полак ЛС, Гольденберг МЯ, Левицкий АА, 1984. Вычислительные методы в химической кинетике. Наука: Москва: 280 с. [Polak LS, Gol'denberg MYa, Levitskiy AA, 1984. Vychislitel'nye metody v khimicheskoy kinetike. Nauka, Moscow: 280 pp. (In Russian)].

31. Попов ФС, 1995. Вычислительные методы инженерной геокриологии. Наука, Новосибирск: 136 с. [Ророv FS, 1995. Vychislitel'nye Metody Inzhenernoy Geokriologii. Nauka, Novosibirsk: 136 pp. (In Russian)].

32. Растворова ОГ, 1983. Физика почв (Практическое руководство). Изд-во Ленингр. ун-та, Ленинград: 196 с. [Rastvorova OG, 1983. Fizika Pochv (Prakticheskoe Rukovodstvo). Izd-vo Leningr. un-ta, Leningrad: 196 pp. (In Russian)].

33. Ревут ИБ, 1972. Физика почв. Колос, Ленинград: 368 с. [Revut IB, 1972. Fizika Pochv. Kolos, Leningrad: 368 pp. (In Russian)].

34. Самарский АA, 1983. Теория разностных схем. Наука, Mockвa: 378-407 c. [Samarskiy AA, 1983. Teoriya Raznostnykh Skhem. Nauka, Moscow: 378-407 pp. (In Russian)].

35. Таланова ГИ, 2018. Климат заповедника «Малая Сосьва»: многолетние данные. Динамика окружающей среды и глобальные изменения климата. 9:22-45. [Talanova Gl, 2018. Climate of the reserve "Malaya Sosva": Iong-term material. Environmental dynamics and global climate change. 9:22-45 (In Russian, English Abstract)]. doi: 10.17816/ edgcc8946

36. Федоренко РП, 2008. Введение в вычислительную физику. Издат. Дом «Интеллект», Долгопрудный: 504 с. [Fedorenko RP, 2008. Vvedenie v Vychislitel'nuyu Fiziku. Izdat. Dom «Intellekt», Dolgoprudnyy: 504 pp. (In Russian)].

37. Федотов ГН, Жуков ДВ, 2004. Органоминеральные гели и температуропроводность почв, с. 114-115 В: Материалы научной сессии по фундаментальному почвоведению: Mосква. [Fedotov GN, Zhukov DV, 2004. Organomineral'nye geli i temperaturoprovodnost' pochv, p. 114-115 In: Materialy nauchnoy sessii po fundamental'nomu pochvovedeniyu, Moscow (In Russian)].

38. Чудновский АФ, 1976. Теплофизика почв. Наука, Москва: 352 с. [Chudnovskiy AF, 1976. Teplofizika Pochv. Nauka, Moscow: 352 pp. (In Russian)].

39. Шеин ЕВ, 2005. Курс физики почв. Изд-во МГУ, Москва: 432 c. [Shein EV, 2005. Kurs Fiziki Pochv. Izd-vo MGU., Moscow: 432 pp. (In Russian)].

40. Шеин ЕВ, Рыжова ИМ, 2016. Математическое моделирование в почвоведении. «ИП Маракушев А.Б», Москва: 377 c. [Shein EV, Ryzhova IM, 2016. Matematicheskoe Modelirovanie v Pochvovedenii. IP Marakushev A.B, Moscow: 377 pp. (In Russian)].

41. Bowden JW, Posner AM, Quirk JP, 1974. A model for ion adsorption on variable charge surfaces, p. 29-34 In: Transactions of the 10th International Congress of Soil Science. V. II., Moscow: Nauka.
42. Brady JB, 1995. Diffusion Data for Silicate Minerals, Glasses, and Liquids, p. 269-290 In: Mineral Physics and Crystallography: A Handbook of Physical Constants, Washington: American Geophysical Union.

43. Costesèque $P$, Mojtabi $A$, Platten JK, 2011. Thermodiffusion phenomena. Comptes Rendus Mécanique. 339:275-279.

44. Diaconis P, Efron B, 1983. Computer-Intensive Methods in Statistics. Scientific American. 248:116-131.

45. Farlow SJ, 1982. Partial Differential Equations for Scientists and Engineers. John Wiley \& Sons, Inc., New York: 448 pp.

46. Glagolev MV, Sabrekov AF, 2019. On several ill-posed and ill-conditioned mathematical problems of soil physics. IOP Conference Series: Earth and Environmental Science. 368:012011.

47. Grew KE, Ibbs TL, 1952. Thermal Diffusion in Gases. Cambridge University Press, Cambridge: 143 pp.

48. Groenevelt PH, 1974. On thermo-osmosis and thermofiltration in porous media, p. 47-55 In: Transactions of the 10th International Congress of Soil Science. V. I., Moscow: Nauka.

49. Hansen PC, 2010. Discrete Inverse Problems: Insight and Algorithms. SIAM, Philadelphia: 213 pp.

50. Jury WA, Gardner WR, Gardner WH, 1991. Soil Physics. John Wiley \& Sons, Inc., New York: 328 pp.

51. Lettau B, 1971. Determination of the Thermal Diffusivity in the Upper Layers of a Natural Ground Cover. Soil Science 112:173-177.

52. Mochenov SYu, Churkina Al, Sabrekov AF, Glagolev MV, Ilyasov DV, Terentieva IE, Maksyutov ShSh, 2018. Soils in seasonally flooded forests as methane sources: A case study of West Siberian South taiga. IOP Conference Series: Earth and Environmental Science. 138:012012.

53. Panikov NS, Blagodatsky SA, Blagodatskaya JV, Glagolev MV, 1992. Determination of microbial mineralization activity in soil by modified Wright and Hobbie method. Biology and Fertility of Soils. 14:280-287.

54. Rice JR, 1981. Matrix Computations and Mathematical Software. McGraw-Hill Book Company, New York: 248 pp.

55. Sabrekov AF, Glagolev MV, Kleptsova I, Machida T, Maksyutov SS, 2013. Methane Emission from Mires of the West Siberian Taiga. Eurasian Soil Science 46:1182-1193.

56. Schechter RS, 1967. The Variational Method in Engineering. McGraw-Hill Book Company, New York: 287 pp.

57. Shih T-M, 1984. Numerical Heat Transfer. Hemisphere Publishing Corporation, Washington: 563 pp.

58. Tikhonov AN, Arsenin VY, 1977. Solutions of III-Posed Problems. Winston \& Sons, Washington: 258 pp.

59. Van Wijk WR, editor, 1963. Physics of Plant Environment. North-Holland Publishing Company, Amsterdam: 382 pp.

60. Zhdanov MS, 2002. Geophysical Inverse Theory and Regularization Problems. Elsevier, Amsterdam: 633 pp.

61. Zhu X, Zhuang Q, Qin Z, Song L, Glagolev M, 2013. Estimating wetland methane emissions from the northern high latitudes from 1990 to 2009 using artificial neural networks. Global Biogeochemical Cycles 27:592-604. 